\title{
Numerical Simulations For Solving FuzzY Fractional DifFERENTIAL EQUATIONS BY MAX-Min IMPROved Euler MeTHODS
}

\author{
Najeeb Alam Khan, Oyoon Abdul Razzaq, Fatima Riaz \\ Department of Mathematical Sciences \\ University of Karachi, Karachi 75270, Pakistan \\ njbalam@yahoo.com
}

\begin{abstract}
In this paper, an extension is introduced into Max-Min Improved Euler methods for solving initial value problems of fuzzy fractional differential equations (FFDEs). Two modified fractional Euler type methods have been proposed and investigated to obtain numerical solutions of linear and nonlinear FFDEs. The proposed algorithms are tested on various illustrative examples. Exact values are also simulated to compare and discuss the closeness and accuracy of approximations so obtained. Comparatively, tables and graphs results reveal the complete reliability, efficiency and accuracy of the proposed methods.
\end{abstract}

Key words: fuzzy, improved Euler method, numerical solution, Mathematica 10

\section{Introduction}

Generalization of ordinary calculus and an important branch in mathematical analysis, "fractional calculus" has become a subject of interest among mathematicians, physicists, and engineers in last few decades [1-5]. Its extensive development and influence in many areas occurred after the invention of differential calculus by Leibniz and Newton. Modeling of physical phenomenon in fractional differential equations (FDEs), although, a difficult task nonetheless has demonstrated applications in diverse fields of science. Recently, problems in many areas like diffusion process, rheology, electrochemistry, viscoelasticity, etc., have been developed and formulated in terms of fractional derivatives and fractional integrals, for instance, time-space fractional diffusion equation models, structural damping models, acoustical wave equations for complex media, fractional Schrodinger equation in quantum theory, etc. [6$10]$. 
Whenever a real-world problem is converted to an ordinary differential equation, sometimes it cannot readily or rapidly be solved by a traditional mathematical method and a numerical method is usually sought and carried out. Correspondingly to ordinary differential equations (ODEs), the exact analytical solutions of fractional differential equations are often difficult, and sometimes impossible to obtain; thus numerical-analytical methods for solving fractional differential equations are of particular importance [11-20].

Fractional differential equations of physical phenomenon are said to be modeled perfectly if every uncertainty is coped out. Therefore, it is necessary to have other theories along with, which would handle issues of uncertainty while modeling. Various theories exist for describing such situations and the most popular among them is fuzzy set theory [21-22]. On contribution of this theory more realistic models have been obtained. Over the last few years, the theoretical framework of fractional differential equations with fuzzy theory has been an on go research field. Fuzzy fractional differential equation is generalization of fuzzy differential equation, which is initially introduced by Agarwal et al. [23] in which he considered differential equation of fractional order with uncertainty and presented the concept of solution. This initiative motivated several researchers to establish some results on the existence and uniqueness of the solutions, for instance, fuzzy Laplace transform, fractional Euler method, iterative techniques etc. are being employed for this purpose [24-28].

In the present paper, an interpretation for fuzzy fractional differential equations has been proposed. Essentially, this work is generalization and extension of Method 1 and Method 2 Improved Euler Method in paper of Smita and Chakraverty [29] to fractional order. Before Smita and Chakraverty, many other authors have made considerable efforts to improve an Euler method, and using it as the stepping-stone of numerical methods for solving initial value problems in differential equations. Basically, this method was introduced by Euler in 1728, which was moreover improved and improved by Heun. Further, Abraham et al. [30] proposed a new improvement on Euler method. Ma et al. [31] used standard Euler approximation method for linear and nonlinear first order fuzzy differential equation. Improved fractional Euler method for fuzzy fractional initial value problem is illustrated by Mazandarani et al. [32]. Ahmad et al. [33] proposed a new fuzzification of the classical Euler method for solution of linear and nonlinear fuzzy differential equations. Allahviranaloo [34] and Shokri [35] have employed iterative solution of improved Euler method for numerical solution of fuzzy differential equations. Duraisamy and Usha[36] used improved Euler method on fuzzy ordinary differential equations. Odibat [37] achieved his goal of numerical solution of linear and nonlinear equations of fractional order by using algorithm based on improved trapezoidal rule and the fractional Euler's method. At this note, two methods of improved fractional Euler methods, Max-Min IFEM and Average IFEM, 
have been illustrated. The approaches are demonstrated by applying them on various linear and nonlinear examples. Obtained numerical values are tabulated for different step size and fuzziness. Further, Graphical representation and their discussions are also presented. And lastly, conclusion is drawn from numerical investigation and comparisons.

\section{Importance of media independent handover IEEE 802.21}

In this section, several basic concepts of Riemann -Liouville integral and Caputo fractional derivative are recalled, which are used throughout this article.

\section{Definition 2.1}

The Riemann-Liouville integral of order $\alpha>0$ for a function $f$ is defined by [38]

$$
J^{\alpha} f(x)=\int_{0}^{x} \frac{(x-\psi)^{\alpha-1}}{\Gamma(\alpha)} f(\psi) d \psi, \quad x>0
$$

Lemma 2.1. Let $f(x)$ be a crisp continuous function and $[\beta\rceil$ - times differentiable in the independent variable $x$ over the interval of differentiation (integration) $[0, x]$. Then the following relation is hold [3].

$$
{ }^{c} D^{\beta} f(x)={ }^{R L} D^{\beta}\left(f(x)-\sum_{k=0}^{\lfloor\beta} \frac{x^{k}}{k !} f_{0}^{(k)}\right) \quad \beta \in(n-1, n), \quad n \in N
$$

where, $f_{0}^{(k)}=\left.\frac{d^{k} f(x)}{d x^{k}}\right|_{x=0}$ and ${ }^{c} D^{\beta}$ are the Caputo derivative operators, $\lfloor\beta\rfloor$ and $[\beta]$ are just the values of $\beta$ rounded up and down to the nearest integer number respectively. ${ }^{R L} D^{\beta}$ is the more common Riemann-Liouville fractional derivative operator which can be defined as follows:

$$
{ }^{R L} D^{\beta} f(x)=\frac{1}{\Gamma(\lceil\beta\rceil-\beta)} \frac{d^{\lceil\beta\rceil}}{d x^{\lceil\beta\rceil}} \int_{0}^{x} \frac{f(\psi)}{(x-\psi)^{1-\beta\rceil+\beta}} d \psi
$$




\section{Fuzzy Number}

Fuzzy number can be defined as a fuzzy set on $R^{n}$ which is upper semicontinuous, convex, normal and compactly supported in a metric space denoted by $E^{1}$. Also an arbitrary fuzzy number can be represented by an ordered pair of lower and upper bound in which lower bound is left-continuous nondecreasing and upper bound is non-increasing functions over the interval $[0,1]$.

\subsection{Trapezoidal Fuzzy Number (TrFN)}

A trapezoidal fuzzy number is defined as $A=\left(b_{1}, b_{2}, b_{3}, b_{4}\right)$ such that $b_{1}<b_{2}<b_{3}<b_{4}$ are four elements of a fuzzy set on $R=(-\infty, \infty)$. Its membership function $\mu A(x)$ is defined as follows:

$$
\mu A(x)=\left\{\begin{array}{l}
\frac{x-b_{1}}{b_{2}-b_{1}}, \text { if } b_{1} \leq x \leq b_{2} \\
1, \quad \text { if } b_{2} \leq x \leq b_{3} \\
\frac{b_{4}-x}{b_{4}-b_{3}}, \text { if } b_{3} \leq x \leq b_{4} \\
0, \quad \text { otherwise }
\end{array}\right.
$$

Though an $r$-cut approach trapezoidal fuzzy number can be represented as an ordered pair, i.e., $\left[\left(b_{2}-b_{1}\right) r+b_{1},-\left(b_{4}-b_{3}\right) r+b_{4}\right]$, where $r \in[0,1]$.

\subsection{Triangular Fuzzy Number (TFN)}

A triangular fuzzy number $B$ is defined as $B=\left(b_{1}, b_{2}, b_{3}\right)$ such that $b_{1}<b_{2}<b_{3}$ are three elements of a fuzzy set on $R=(-\infty, \infty)$. Its membership function $\mu B(x)$ is defined as follows: 


$$
\mu B(x)=\left\{\begin{array}{l}
\frac{x-b_{1}}{b_{2}-b_{1}}, \text { if } b_{1} \leq x \leq b_{2} \\
\frac{b_{3}-x}{b_{3}-b_{2}}, \text { if } b_{2} \leq x \leq b_{3} \\
0, \quad \text { otherwise }
\end{array}\right.
$$

Definition of fuzzy initial value problem can be found in [29].

\section{Fuzzy fractional differential equations}

Consider the following nth order FFDE by

$$
\begin{gathered}
D_{x}^{n \beta} y(x)+g\left(x, y(x), D_{x}^{\beta} y(x), \ldots\right)=f\left(x, y(x), D_{x}^{\beta} y(x), \ldots\right) \\
y\left(x_{0}\right)=y_{0}, y^{\prime}\left(x_{0}\right)=y_{0}^{\prime}
\end{gathered}
$$

where $y(x)=(y(x, r), \bar{y}(x, r))$ is a fuzzy function of $x$, $f\left(x, y(x), D_{x}^{\beta} y(x), \ldots\right)$ and $g\left(x, y(x), D_{x}^{\beta} y(x), \ldots\right)$ are linear fuzzy-valued functions.

The generalized fractional Euler's method that has been formulated for the numerical solution of initial value problems with Caputo derivatives is presented by Odibat and Momani [37].

Suppose that $D_{x}^{k \beta} f(x) \in C(0, a]$, for $k=0,1,2, \ldots, n+1$, where $0<\beta \leq 1$ Then we have

$$
f(x)=\sum_{i=o}^{n} \frac{x^{i \beta}}{\Gamma(i \beta+1)}\left(D^{i \beta}\right)(0+)+\frac{\left(D^{(n+1) \beta} f\right)(\zeta)}{\Gamma((n+1) \beta+1)} x^{(n+1) \beta},
$$

With $0 \leq \zeta \leq x, \forall x \in(0, a)$.

In case $\beta=1$, the generalized Taylor's formula in Eq. (8) reduces to the classical Taylor's formula. With the aim to find the high accuracy for solving fuzzy fractional differential equations, the initial value problem is considered as: 


$$
{ }^{c} D_{x}^{\beta} \widetilde{y}_{n}(x)=f\left(x_{n}, \widetilde{y}_{n}\left(x_{n}\right)\right), \quad \tilde{y}(0)=\widetilde{y}_{0}, \quad 0<\beta \leq 1, \quad x_{n}>0
$$

The fuzzy fractional initial value problem (FFIVP) can be considered equivalently by the following initial value problems

$$
\begin{gathered}
{ }^{c} D_{x}^{\beta} \underline{y}_{n}^{r}(x)=\left\lfloor f\left(x_{n}, \underline{y}_{n}\left(x_{n}\right)\right)\right]=F\left(x_{n}, \bar{y}_{n}^{r}\left(x_{n}\right), \underline{y}_{n}^{r}\left(x_{n}\right)\right) \\
{ }^{c} D_{x}^{\beta} \bar{y}_{n}^{r}(x)=\left[f\left(x_{n}, \bar{y}_{n}\left(x_{n}\right)\right)\right]=G\left(x_{n}, \bar{y}_{n}^{r}\left(x_{n}\right), \underline{y}_{n}^{r}\left(x_{n}\right)\right) \\
\bar{y}^{r}(0)=\bar{y}_{0}^{r}, \quad \underline{y}^{r}(0)=\underline{y}_{0}^{r}
\end{gathered}
$$

Let $[0, a]$ be the interval over which the solution of the problem is needed. The focus is not to acquire a function $y(x)$ that satisfies the initial value problem. Alternately, an approximation has been made with the help of a set of points $\left\{\left(x_{j}, y\left(x_{j}\right)\right)\right\}$. Using Taylor expansion:

$\underline{y}_{n+1}\left(x_{n+1}\right)=\underline{y}_{n}\left(x_{n}\right)+\frac{h^{\beta}}{\Gamma(\beta+1)}\left({ }^{c} D_{x}^{\beta} \underline{y}_{n}\left(x_{n}\right)\right)+\frac{h^{2 \beta}}{\Gamma(2 \beta+1)}\left({ }^{c} D_{x}^{2 \beta} \underline{y}_{n}\left(x_{n}\right)\right)+\frac{h^{3 \beta}}{\Gamma(3 \beta+1)}\left({ }^{c} D_{x}^{3 \beta} \underline{y}_{n}\left(x_{n}\right)\right)$

and

$\bar{y}_{n+1}\left(x_{n+1}\right)=\bar{y}_{n}\left(x_{n}\right)+\frac{h^{\beta}}{\Gamma(\beta+1)}\left({ }^{c} D_{x}^{\beta} \bar{y}_{n}\left(x_{n}\right)\right)+\frac{h^{2 \beta}}{\Gamma(2 \beta+1)}\left({ }^{c} D_{x}^{2 \beta} \bar{y}_{n}\left(x_{n}\right)\right)+\frac{h^{3 \beta}}{\Gamma(3 \beta+1)}\left({ }^{c} D_{x}^{3 \beta} \bar{y}_{n}\left(x_{n}\right)\right)$

Since

$$
{ }^{c} D_{x}^{2 \beta} \underline{y}_{n}\left(x_{n}\right)=\Gamma(\beta+1)\left(\frac{{ }^{c} D_{x}^{\beta} \underline{y}_{n+1}\left(x_{n+1}\right){ }^{c} D_{x}^{\beta} \underline{y}_{n}\left(x_{n}\right)}{h^{\beta}}\right)
$$

and

$$
{ }^{c} D_{x}^{2 \beta} \bar{y}_{n}\left(x_{n}\right)=\Gamma(\beta+1)\left(\frac{{ }^{c} D_{x}^{\beta} \bar{y}_{n+1}\left(x_{n+1}\right)-{ }^{c} D_{x}^{\beta} \bar{y}_{n}\left(x_{n}\right)}{h^{\beta}}\right)
$$

Therefore, Eqs. (12)-(13) becomes,

$$
\underline{y}_{n+1}\left(x_{n+1}\right)=\underline{y}_{n}\left(x_{n}\right)+\frac{h^{\beta}}{\Gamma(\beta+1)}{ }^{c} D_{x}^{\beta} \underline{y}_{n}\left(x_{n}\right)+\frac{h^{2 \beta}}{\Gamma(2 \beta+1)}\left(\Gamma(\beta+1)\left(\frac{\left.{ }^{c} D_{x}^{\beta} \underline{y}_{n+1}\left(x_{n+1}\right)\right)^{c} D_{x}^{\beta} \underline{y}_{n}\left(x_{n}\right)}{h^{\beta}}\right)\right)
$$

and 


$$
\bar{y}_{n+1}\left(x_{n+1}\right)=\bar{y}_{n}\left(x_{n}\right)+\frac{h^{\beta}}{\Gamma(\beta+1)}{ }^{c} D_{x}^{\beta} \bar{y}_{n}\left(x_{n}\right)+\frac{h^{2 \beta}}{\Gamma(2 \beta+1)}\left(\Gamma(\beta+1)\left(\frac{\left.{ }^{c} D_{x}^{\beta} \bar{y}_{n+1}\left(x_{n+1}\right)\right)^{c} D_{x}^{\beta} \bar{y}_{n}\left(x_{n}\right)}{h^{\beta}}\right)\right)
$$

Implies

$\underline{y}_{n+1}\left(x_{n+1}\right)=\underline{y}_{n}\left(x_{n}\right)+h^{\beta}\left(\frac{\Gamma(2 \beta+1)-(\Gamma(\beta+1))^{2}}{(\Gamma(\beta+1))(\Gamma(2 \beta+1))}\right)^{c} D_{x}^{\beta} \underline{y}_{n}\left(x_{n}\right)+\frac{h^{\beta}(\Gamma(\beta+1)) c_{c}}{\Gamma(2 \beta+1)} D_{x}^{\beta} \underline{y}_{n+1}\left(x_{n+1}\right)$

and

$\bar{y}_{n+1}\left(x_{n+1}\right)=\bar{y}_{n}\left(x_{n}\right)+h^{\beta}\left(\frac{\Gamma(2 \beta+1)-(\Gamma(\beta+1))^{2}}{(\Gamma(\beta+1))(\Gamma(2 \beta+1))}\right)^{c} D_{x}^{\beta} \bar{y}_{n}\left(x_{n}\right)+\frac{h^{\beta}(\Gamma(\beta+1))}{\Gamma(2 \beta+1)} D_{x}^{\beta} \bar{y}_{n+1}\left(x_{n+1}\right)$

when

$$
\begin{gathered}
{ }^{c} D_{x}^{\beta} \bar{y}_{n}\left(x_{n}\right)=\bar{f}\left(x_{n}, y_{n}\left(x_{n}\right)\right),{ }^{c} D_{x}^{\beta} \underline{y}_{n}\left(x_{n}\right)=\underline{f}\left(x_{n}, y_{n}\left(x_{n}\right)\right), \\
{ }^{c} D_{x}^{\beta} \bar{y}_{n+1}\left(x_{n+1}\right)=\bar{f}\left(x_{n+1}, y_{n+1}\left(x_{n+1}\right)\right)
\end{gathered}
$$

and

$$
{ }^{c} D_{x}^{\beta} \underline{y}_{n+1}\left(x_{n+1}\right)=\underline{f}\left(x_{n+1}, y_{n+1}\left(x_{n+1}\right)\right)
$$

are substituted into equations (16) and (17), consequently an expression for $\underline{y}_{n+1}, \bar{y}_{n+1}$ is obtained:

$$
\underline{y}_{n+1}\left(x_{n+1}\right)=\underline{y}_{n}\left(x_{n}\right)+h^{\beta}\left(\frac{\Gamma(2 \beta+1)-(\Gamma(\beta+1))^{2}}{(\Gamma(\beta+1))(\Gamma(2 \beta+1))}\right) \underline{f}\left(x_{n}, y_{n}\left(x_{n}\right)\right)+\frac{h^{\beta}(\Gamma(\beta+1))}{\Gamma(2 \beta+1)} \underline{f}^{\left(x_{n+1}, y_{n+1}\left(x_{n+1}\right)\right)}
$$

and

$$
\bar{y}_{n+1}\left(x_{n+1}\right)=\bar{y}_{n}\left(x_{n}\right)+h^{\beta}\left(\frac{\Gamma(2 \beta+1)-(\Gamma(\beta+1))^{2}}{(\Gamma(\beta+1))(\Gamma(2 \beta+1))}\right) \bar{f}\left(x_{n}, y_{n}\left(x_{n}\right)\right)+\frac{h^{\beta}(\Gamma(\beta+1))}{\Gamma(2 \beta+1)} \bar{f}\left(x_{n+1}, y_{n+1}\left(x_{n+1}\right)\right)
$$

A system of points that approximate the solution of $y(x)$ is produced by above recursive process and at each step the fractional Euler's method is used as a prediction.

\section{Proposed methods for FFDE}

In this section, two methods to solve FFDE are proposed and illustrated. 


\subsection{Max-Min Modified Fractional Euler Method (Method 1)}

Considering all the possible combination of lower and upper values of the variable and by using modified fractional Euler method we obtain

$$
\begin{aligned}
& \underline{y}_{n+1}^{(1)}\left(x_{n+1} ; r\right)=\underline{y}_{n}+h^{\beta}\left(\frac{\Gamma(2 \beta+1)-(\Gamma(\beta+1))^{2}}{(\Gamma(\beta+1))(\Gamma(2 \beta+1))}\right) f\left(x_{n}, \underline{y}_{n}\left(x_{n} ; r\right)\right)+\frac{h^{\beta}(\Gamma(\beta+1))}{\Gamma(2 \beta+1)} f\left(x_{n+1}, \underline{y}_{n+1}\left(x_{n+1} ; r\right)\right), \\
& \bar{y}_{n+1}^{(1)}\left(x_{n+1} ; r\right)=\bar{y}_{n}+h^{\beta}\left(\frac{\Gamma(2 \beta+1)-(\Gamma(\beta+1))^{2}}{(\Gamma(\beta+1))(\Gamma(2 \beta+1))}\right) f\left(x_{n}, \bar{y}_{n}\left(x_{n} ; r\right)\right)+\frac{h^{\beta}(\Gamma(\beta+1))}{\Gamma(2 \beta+1)} f\left(x_{n+1}, \bar{y}_{n+1}\left(x_{n+1} ; r\right)\right), \\
& \underline{y}_{n+1}^{(2)}\left(x_{n+1} ; r\right)=\underline{y}_{n}+h^{\beta}\left(\frac{\Gamma(2 \beta+1)-(\Gamma(\beta+1))^{2}}{(\Gamma(\beta+1))(\Gamma(2 \beta+1))}\right) f\left(x_{n}, \bar{y}_{n}\left(x_{n} ; r\right)\right)+\frac{h^{\beta}(\Gamma(\beta+1))}{\Gamma(2 \beta+1)} f\left(x_{n+1}, \bar{y}_{n+1}\left(x_{n+1} ; r\right)\right), \\
& \bar{y}_{n+1}^{(2)}\left(x_{n+1} ; r\right)=\bar{y}_{n}+h^{\beta}\left(\frac{\Gamma(2 \beta+1)-(\Gamma(\beta+1))^{2}}{(\Gamma(\beta+1))(\Gamma(2 \beta+1))}\right) f\left(x_{n}, \underline{y}_{n}\left(x_{n} ; r\right)\right)+\frac{h^{\beta}(\Gamma(\beta+1))}{\Gamma(2 \beta+1)} f\left(x_{n+1}, \underline{y}_{n+1}\left(x_{n+1} ; r\right)\right) .
\end{aligned}
$$

The exact and approximate solution to $x_{n} ; 0 \leq n \leq N$ are denoted by $\left[Y_{n}\left(x_{n} ; r\right)\right]=\left\lfloor\left(\underline{Y}\left(x_{n} ; r\right), \bar{Y}\left(x_{n} ; r\right)\right)\right]$ and $\left[y_{n}\left(x_{n} ; r\right)\right]=\left[\left(\underline{y}\left(x_{n} ; r\right), \bar{y}\left(x_{n} ; r\right)\right)\right], \quad$ respectively. However, minimum and maximum are taken from Eqs. (18)-(21) for lower and upper values of the independent variable $x_{n}$.

$$
\begin{aligned}
& \underline{y}_{n+1}\left(x_{n+1} ; r\right)=\min \left\{\underline{y}_{n+1}^{(1)}\left(x_{n+1} ; r\right), \underline{y}_{n+1}^{(2)}\left(x_{n+1} ; r\right)\right\} \\
& \bar{y}_{n+1}\left(x_{n+1} ; r\right)=\max \left\{\hat{y}_{n+1}^{(1)}\left(x_{n+1} ; r\right), \bar{y}_{n+1}^{(2)}\left(x_{n+1} ; r\right)\right\}
\end{aligned}
$$

The above procedure leads to the better approximations to the exact solutions. The efficiency and powerfulness of the methodology are demonstrated by variety of examples.

\subsection{Average Modified Fractional Euler Method (Method 2)}

In the second method, similarly averages of lower and upper values are computed respectively. Then the Eqs. (18)-(21) reduces to 


$$
\begin{aligned}
& \underline{y}_{n+1}=\frac{1}{2}\left[\underline{y}^{(1)}\left(x_{n+1} ; r\right)+\underline{y}^{(2)}\left(x_{n+1} ; r\right)\right] \\
& \bar{y}_{n+1}=\frac{1}{2}\left[\bar{y}^{(1)}\left(x_{n+1} ; r\right)+\bar{y}^{(2)}\left(x_{n+1} ; r\right)\right]
\end{aligned}
$$

\section{Numerical problems}

\section{Problem 1}

Let us consider a linear triangular FIVP given in [29] for fractional order.

$$
D_{x}^{\beta} y(x)=y(x), y(0)=(0.75,1,1.25) \text {. }
$$

Then using the r-cut approach, the triangular fuzzy initial condition can be represented as

$$
y(0)=[0.75+0.25 r, 1.125-0.125 r], 0 \leq r \leq 1 .
$$

The results of problem 1, obtained by the above Method 1 and Method 2 are tabulated in Table 1and 2 for different values of.

Table 1. Comparison between Exact and proposed methods of Problem 1 for $x=1$,

$$
h=0.001, \beta=1
$$

\begin{tabular}{|l|l|l|l|}
\hline $\mathrm{r}$ & Exact values & \multicolumn{2}{|l|}{ Modified Fractional Euler Methods } \\
\cline { 3 - 4 } & & Method 1 & Method 2 \\
& & $\beta=1$ & $\beta=1$ \\
\hline 0 & {$[2.03871,3.05807]$} & {$[2.03871,3.05807]$} & {$[2.03922,3.05756]$} \\
\hline 0.2 & {$[2.17463,2.99011]$} & {$[2.17463,2.99011]$} & {$[2.17503,2.9897]$} \\
\hline 0.4 & {$[2.31054,2.92215]$} & {$[2.31054,2.92215]$} & {$[2.31084,2.92185]$} \\
\hline 0.6 & {$[2.44645,2.8542]$} & {$[2.44645,2.8542]$} & {$[2.44666,2.85399]$} \\
\hline 0.8 & {$[2.58237,2.78624]$} & {$[2.58237,2.78624]$} & {$[2.58247,2.78614]$} \\
\hline 1 & {$[2.71828,2.71828]$} & {$[2.71828,2.71828]$} & {$[2.71828,2.71828]$} \\
\hline
\end{tabular}


Table 2. Results obtained for Problem 1 by Method 1 and Method 2 for $x=1$, $h=0.1, \beta=0.75$ and $\beta=0.85$

\begin{tabular}{|l|l|l|l|l|}
\hline \multicolumn{5}{|c|}{ Modified Fractional Euler Methods } \\
\hline $\mathrm{r}$ & Method 1 & Method 2 & Method 1 & Method 2 \\
& $\beta=0.75$ & $\beta=0.75$ & $\beta=0.85$ & $\beta=0.85$ \\
\hline 0 & {$[5.35740,8.03611]$} & {$[5.59647,7.79704]$} & {$[3.37463,5.06194]$} & {$[3.49243,4.94414]$} \\
\hline 0.2 & {$[5.71456,7.85753]$} & {$[5.90582,7.66627]$} & {$[3.59960,4.94945]$} & {$[3.69384,4.85521]$} \\
\hline 0.4 & {$[6.07173,7.67895]$} & {$[6.21516,7.53551]$} & {$[3.82458,4.83696]$} & {$[3.89526,4.76628]$} \\
\hline 0.6 & {$[6.42889,7.50037]$} & {$[6.52451,7.40474]$} & {$[4.04955,4.72448]$} & {$[4.09667,4.67736]$} \\
\hline 0.8 & {$[6.78605,7.32179]$} & {$[6.83386,7.27397]$} & {$[4.27453,4.61199]$} & {$[4.29809,4.58843]$} \\
\hline 1 & {$[7.14321,7.14321]$} & {$[7.14321,7.14321]$} & {$[4.27453,4.4995]$} & {$[4.49950,4.49950]$} \\
\hline
\end{tabular}

\section{Problem 2}

Next consider a linear trapezoidal FIVP given in [29] for fractional order.

$$
D_{x}^{\beta} y(x)=y(x), \quad y(0)=(0.75,0.85,1.1,1.25)
$$

Then using the $r$-cut approach, the trapezoidal fuzzy initial condition can be represented as

$$
y(0)=[0.75+0.1 r, 1.125-0.025 r], 0 \leq r \leq 1
$$

The results of problem 2, obtained by the above Method 1 and Method 2 are tabulated in Table 3 and 4 for different values of $r$.

Table 3. Comparison between Exact and proposed methods of Problem 2 for $x=1$,

$$
h=0.001, \beta=1
$$

\begin{tabular}{|l|l|l|l|}
\hline $\mathrm{r}$ & Exact Values & \multicolumn{2}{|c|}{ Modified Fractional Euler Methods } \\
\cline { 3 - 4 } & & $\begin{array}{l}\text { Method 1 } \\
\beta=1\end{array}$ & $\begin{array}{l}\text { Method } 2 \\
\beta=1\end{array}$ \\
\hline 0 & {$[2.03871,3.05807]$} & {$[2.03871,3.05807]$} & {$[2.03922,3.05756]$} \\
\hline 0.2 & {$[2.09308,3.04448]$} & {$[2.09308,3.04448]$} & {$[2.09355,3.04400]$} \\
\hline 0.4 & {$[2.14744,3.03088]$} & {$[2.14744,3.03088]$} & {$[2.14788,3.03044]$} \\
\hline 0.6 & {$[2.20181,3.01729]$} & {$[2.20181,3.01729]$} & {$[2.20222,3.01688]$} \\
\hline 0.8 & {$[2.25617,3.0037]$} & {$[2.25617,3.0037]$} & {$[2.25655,3.00333]$} \\
\hline 1 & {$[2.31054,2.99011]$} & {$[2.31054,2.99011]$} & {$[2.31088,2.98977]$} \\
\hline
\end{tabular}


Table 4. Results obtained for Problem 2 by Method 1 and Method 2 for $x=1$, $h=0.1, \beta=0.75$ and $\beta=0.85$

\begin{tabular}{|l|l|l|l|l|}
\hline \multicolumn{5}{|c|}{ Modified Fractional Euler Methods } \\
\hline $\mathrm{R}$ & Method 1 & Method 2 & Method 1 & Method 2 \\
& $\beta=0.75$ & $\beta=0.75$ & $\beta=0.85$ & $\beta=0.85$ \\
\hline 0 & {$[5.35740,8.03611]$} & {$[5.59647,7.79704]$} & {$[3.37463,5.06194]$} & {$[3.49243,4.94414]$} \\
\hline 0.2 & {$[5.50027,8.00039]$} & {$[5.72340,7.77726]$} & {$[3.46462,5.03944]$} & {$[3.57457,4.92949]$} \\
\hline 0.4 & {$[5.64313,7.96467]$} & {$[5.85032,7.75748]$} & {$[3.55461,5.01695]$} & {$[3.65670,4.91485]$} \\
\hline 0.6 & {$[5.78600,7.92896]$} & {$[5.97725,7.73771]$} & {$[3.64460,4.99445]$} & {$[3.73884,4.90021]$} \\
\hline 0.8 & {$[5.92886,7.89324]$} & {$[6.10418,7.71793]$} & {$[3.73459,4.97195]$} & {$[3.82098,4.88556]$} \\
\hline 1 & {$[6.07173,7.85753]$} & {$[6.23110,7.69815]$} & {$[3.82458,4.94945]$} & {$[3.90311,4.87092]$} \\
\hline
\end{tabular}

\section{Problem 3}

Consider a nonlinear triangular FIVP for fractional order.

$$
D_{x}^{\beta} y(x)=-y(x)+y(x)^{2}, y(0)=(0.75,1,1.25), 0 \leq r \leq 1
$$

Using the $r$-cut approach, the triangular fuzzy initial condition can be represented as

$$
y(0)=[0.75+0.25 r, 1.125-0.125 r], 0 \leq r \leq 1
$$

Obtained results by Method 1 Method 2 are tabulated in Table 5 and 6 for different values of $r$.

Table 5. Comparison between Exact and proposed methods of Problem 3 for $x=1$,

$$
h=0.001, \beta=1
$$

\begin{tabular}{|l|l|l|l|}
\hline $\mathrm{r}$ & Exact Values & \multicolumn{2}{|c|}{ Modified Fractional Euler Methods } \\
\cline { 3 - 4 } & & $\begin{array}{l}\text { Method 1 } \\
\beta=1\end{array}$ & $\begin{array}{l}\text { Method 2 } \\
\beta=1\end{array}$ \\
\hline 0 & {$[0.524633,1.43273]$} & {$[0.524633,1.43273]$} & {$[0.525068,1.43229]$} \\
\hline 0.2 & {$[0.59539,1.32823]$} & {$[0.59539,1.32823]$} & {$[0.595729,1.32789]$} \\
\hline 0.4 & {$[0.675814,1.23403]$} & {$[0.675814,1.23403]$} & {$[0.676068,1.23378]$} \\
\hline 0.6 & {$[0.768031,1.14869]$} & {$[0.768031,1.14869]$} & {$[0.768205,1.14851]$} \\
\hline 0.8 & {$[0.874839,1.07101]$} & {$[0.874839,1.07101]$} & {$[0.874932,1.07091]$} \\
\hline 1 & {$[1.000000,1.00000]$} & {$[1.000000,1.00000]$} & {$[1.000000,1.00000]$} \\
\hline
\end{tabular}


Table 6. Results obtained for Problem 3 by Method 1 and Method 2 for $x=1$, $h=0.1, \beta=0.75$ and $\beta=0.85$

\begin{tabular}{|l|l|l|l|l|}
\hline \multicolumn{5}{|c|}{ Modified Fractional Euler Methods } \\
\hline $\mathrm{R}$ & Method 1 & Method 2 & Method 1 & $\begin{array}{l}\text { Method 2 } \\
\beta=0.85\end{array}$ \\
& $\beta=0.75$ & $\beta=0.75$ & $\beta=0.85$ & $\beta=10.65]$ \\
\hline 0 & {$[0.34438,2.87449]$} & {$[0.72317,2.49570]$} & {$[0.40220,2.00084]$} & {$[0.54076,1.65376]$} \\
\hline 0.2 & {$[0.36505,2.85525]$} & {$[0.73933,2.48096]$} & {$[0.47238,1.69349]$} & {$[0.56576,1.60011]$} \\
\hline 0.4 & {$[0.44727,2.00193]$} & {$[0.62307,1.82613]$} & {$[0.55867,1.45838]$} & {$[0.62081,1.39625]$} \\
\hline 0.6 & {$[0.56075,1.51888]$} & {$[0.64884,1.43079]$} & {$[0.66742,1.27305]$} & {$[0.70700,1.23346]$} \\
\hline 0.8 & {$[0.72802,1.21165]$} & {$[0.76858,1.17109]$} & {$[0.80876,1.12335]$} & {$[0.82935,1.10276]$} \\
\hline 1 & {$[1.00000,1.00000]$} & {$[1.00000,1.00000]$} & {$[1.00000,1.00000]$} & {$[1.00000,1.00000]$} \\
\hline
\end{tabular}

\section{Problem 4}

Now consider a nonlinear trapezoidal FIVP for fractional order.

$$
D_{x}^{\beta} y(x)=-y(x)+y(x)^{2}, y(0)=(0.75,0.85,1.1,1.25 r), 0 \leq r \leq 1
$$

Using $r$ - cut approach, the trapezoidal fuzzy initial condition can be represented as

$$
y(0)=[0.75+0.1 r, 1.125-0.025 r], 0 \leq r \leq 1
$$

Results of this problem, obtained by Method 1 Method 2 are tabulated in Table 7 and 8 for different values of $r$.

Table 7. Comparison between Exact and proposed methods of Problem 4 for $x=1$,

$$
h=0.001 \text { and } \beta=1
$$

\begin{tabular}{|l|l|l|l|}
\hline $\mathrm{r}$ & Exact Values & \multicolumn{2}{|c|}{ Modified Fractional Euler Methods } \\
\cline { 3 - 4 } & & $\begin{array}{l}\text { Method 1 } \\
\beta=1\end{array}$ & $\begin{array}{l}\text { Method } 2 \\
\beta=1\end{array}$ \\
\hline 0 & {$[0.52463,1.43273]$} & {$[0.52463,1.43273]$} & {$[0.52507,1.43229]$} \\
\hline 0.2 & {$[0.55189,1.41092]$} & {$[0.55189,1.41092]$} & {$[0.55230,1.41051]$} \\
\hline 0.4 & {$[0.58052,1.38959]$} & {$[0.58052,1.38959]$} & {$[0.58092,1.38919]$} \\
\hline 0.6 & {$[0.61064,1.36870]$} & {$[0.61064,1.36870]$} & {$[0.61101,1.36833]$} \\
\hline 0.8 & {$[0.64236,1.34825]$} & {$[0.64236,1.34825]$} & {$[0.64271,1.34790]$} \\
\hline 1 & {$[0.67581,1.32823]$} & {$[0.67581,1.32823]$} & {$[0.67614,1.32790]$} \\
\hline
\end{tabular}


Table 8. Results obtained for Problem 4 by Method 1 and Method 2 for $x=1$, $h=0.1, \beta=0.75$ and $\beta=0.85$

\begin{tabular}{|l|l|l|l|l|}
\hline \multicolumn{5}{|c|}{ Modified Fractional Euler Methods } \\
\hline $\mathrm{r}$ & Method 1 & Method 2 & Method 1 & Method 2 \\
& $\beta=0.75$ & $\beta=0.75$ & $\beta=0.85$ & $\beta=0.85$ \\
\hline 0 & {$[0.34438,2.87449]$} & {$[0.72317,2.49570]$} & {$[0.43847,1.75604]$} & {$[0.54076,1.65376]$} \\
\hline 0.2 & {$[0.32569,4.16151]$} & {$[1.07889,3.40834]$} & {$[0.42864,1.93209]$} & {$[0.55812,1.80261]$} \\
\hline 0.4 & {$[0.35124,3.74846]$} & {$[0.97758,3.12212]$} & {$[0.45721,1.86728]$} & {$[0.57654,1.74796]$} \\
\hline 0.6 & {$[0.37964,3.40168]$} & {$[0.90444,2.87688]$} & {$[0.48819,1.80610]$} & {$[0.59812,1.69617]$} \\
\hline 0.8 & {$[0.41143,3.10741]$} & {$[0.85409,2.66475]$} & {$[0.52188,1.74826]$} & {$[0.62307,1.64707]$} \\
\hline 1 & {$[0.44727,2.85525]$} & {$[0.82280,2.47971]$} & {$[0.55867,1.69349]$} & {$[0.65165,1.60051]$} \\
\hline
\end{tabular}

\section{Problem 5}

Let us consider another nonlinear triangular FIVP, also studied in [29], for fractional order.

$$
D_{x}^{\beta} y(x)=e^{-y(x)^{2}}, y(0)=(0.75,1,1.5), 0 \leq r \leq 1
$$

Usingthe r-cut approach, the triangular fuzzy initial condition can be represented as

$$
y(0)=[0.75+0.25 r, 1.5-0.5 r] \quad, 0 \leq r \leq 1 .
$$

Outcomes so obtained by Method 1 Method 2 are tabulated in Table 9 and 10 for different values of $r$.

Table 9. Comparison between Exact and proposed methods of Problem 5 for $x=1$,

$$
h=0.001, \beta=1
$$

\begin{tabular}{|l|l|l|l|}
\hline $\mathrm{r}$ & Exact Values & \multicolumn{2}{|c|}{ Modified Fractional Euler Methods } \\
\cline { 3 - 4 } & & $\begin{array}{l}\text { Method 1 } \\
\beta=1\end{array}$ & $\begin{array}{l}\text { Method 2 } \\
\beta=1\end{array}$ \\
\hline 0 & {$[1.14353,1.59134]$} & {$[1.14334,1.59154]$} & {$[1.14343,1.59144]$} \\
\hline 0.2 & {$[1.16752,1.51823]$} & {$[1.16736,1.51838]$} & {$[1.16744,1.51831]$} \\
\hline 0.4 & {$[1.19208,1.4497]$} & {$[1.19196,1.44982]$} & {$[1.19202,1.44976]$} \\
\hline 0.6 & {$[1.21727,1.38565]$} & {$[1.21719,1.38573]$} & {$[1.21723,1.38569]$} \\
\hline 0.8 & {$[1.24318,1.32584]$} & {$[1.24314,1.32588]$} & {$[1.24316,1.32586]$} \\
\hline 1 & {$[1.26987,1.26987]$} & {$[1.26987,1.26987]$} & {$[1.26987,1.26987]$} \\
\hline
\end{tabular}


Table 10. Results obtained for Problem 5 by Method 1 and Method 2 for $x=1$, $h=0.1, \beta=0.75$ and $\beta=0.85$

\begin{tabular}{|l|l|l|l|l|}
\hline \multicolumn{5}{|c|}{ Modified Fractional Euler Methods } \\
\hline $\mathrm{r}$ & Method 1 & Method 2 & Method 1 & Method 2 \\
& $\beta=0.75$ & $\beta=0.75$ & $\beta=0.85$ & $\beta=0.85$ \\
\hline 0 & {$[1.31283,1.67807]$} & {$[1.32335,1.66755]$} & {$[1.23527,1.64905]$} & {$[1.24582,1.63849]$} \\
\hline 0.2 & {$[1.33222,1.61581]$} & {$[1.34072,1.60731]$} & {$[1.25774,1.58069]$} & {$[1.26631,1.57212]$} \\
\hline 0.4 & {$[1.35233,1.55901]$} & {$[1.35874,1.55261]$} & {$[1.28098,1.51748]$} & {$[1.28748,1.51097]$} \\
\hline 0.6 & {$[1.37317,1.5073]$} & {$[1.37744,1.50302]$} & {$[1.30502,1.45916]$} & {$[1.30939,1.45479]$} \\
\hline 0.8 & {$[1.39474,1.46018]$} & {$[1.39688,1.45804]$} & {$[1.32989,1.40536]$} & {$[1.33208,1.40316]$} \\
\hline 1 & {$[1.41710,1.41710]$} & {$[1.41710,1.41710]$} & {$[1.35560,1.35560]$} & {$[1.35560,1.35560]$} \\
\hline
\end{tabular}

\section{Problem 6}

Now consider nonlinear trapezoidal FIVP for fractional order.

$$
D_{x}^{\beta} y(x)=e^{-x^{2}} y(0)=(0.75,0.85,1.3,1.5) 0 \leq r \leq 1
$$

Using $r$ - cut approach, the trapezoidal fuzzy initial condition can be represented as

$$
y(0)=[0.75+0.1 r, 1.5-0.2 r], 0 \leq r \leq 1
$$

Results so obtained by Method 1 Method 2 are tabulated in Table 11 and 12 for different values of $r$.

Table 11. Comparison between Exact and proposed methods of Problem 6 for $x=1$,

$$
h=0.001 \text { and } \beta=1 \text {. }
$$

\begin{tabular}{|l|l|l|l|}
\hline $\mathrm{r}$ & Exact Values & \multicolumn{2}{|c|}{ Modified Fractional Euler Methods } \\
\cline { 3 - 4 } & & $\begin{array}{l}\text { Method 1 } \\
\beta=1\end{array}$ & $\begin{array}{l}\text { Method 2 } \\
\beta=1\end{array}$ \\
\hline 0 & {$[1.14353,1.59134]$} & {$[1.14334,1.59154]$} & {$[1.14343,1.59144]$} \\
\hline 0.2 & {$[1.15306,1.56155]$} & {$[1.15288,1.56173]$} & {$[1.15297,1.56164]$} \\
\hline 0.4 & {$[1.16268,1.53248]$} & {$[1.16252,1.53265]$} & {$[1.16260,1.53257]$} \\
\hline 0.6 & {$[1.17238,1.50416]$} & {$[1.17223,1.50430]$} & {$[1.17231,1.50423]$} \\
\hline 0.8 & {$[1.18218,1.47656]$} & {$[1.18205,1.47670]$} & {$[1.18211,1.47663]$} \\
\hline 1 & {$[1.19208,1.44970]$} & {$[1.19196,1.44982]$} & {$[1.19202,1.44976]$} \\
\hline
\end{tabular}


Table 12. Results obtained for Problem 6 by Method 1 and Method 2 for $x=1$,

$$
h=0.1, \beta=0.75 \text { and } \beta=0.85
$$

\begin{tabular}{|l|l|l|l|l|}
\hline \multicolumn{5}{|c|}{ Modified Fractional Euler Methods } \\
\hline $\mathrm{r}$ & Method 1 & Method 2 & Method 1 & Method 2 \\
& $\beta=0.75$ & $\beta=0.75$ & $\beta=0.85$ & $\beta=0.85$ \\
\hline 0 & {$[1.31283,1.67807]$} & {$[1.32335,1.66755]$} & {$[1.23527,1.64905]$} & {$[1.24582,1.63849]$} \\
\hline 0.2 & {$[1.3205,1.65249]$} & {$[1.33022,1.64277]$} & {$[1.24416,1.62108]$} & {$[1.25394,1.61131]$} \\
\hline 0.4 & {$[1.32829,1.62782]$} & {$[1.33719,1.61891]$} & {$[1.25318,1.59395]$} & {$[1.26216,1.58497]$} \\
\hline 0.6 & {$[1.33619,1.60402]$} & {$[1.34427,1.59594]$} & {$[1.26232,1.56765]$} & {$[1.27049,1.55948]$} \\
\hline 0.8 & {$[1.3442,1.5811]$} & {$[1.35145,1.57385]$} & {$[1.27159,1.54216]$} & {$[1.27893,1.53482]$} \\
\hline 1 & {$[1.35233,1.55901]$} & {$[1.35874,1.55261]$} & {$[1.28098,1.51748]$} & {$[1.28748,1.51097]$} \\
\hline
\end{tabular}

\section{Results and discussion}

In this section, corresponding plots of problems 1-6 are given for different values of $r$. All the figures represent the comparison between exact and approximated values obtained by Method 1 and Method 2. Triangular curves in figures 1-2, 5-6 and 9-10 verifies the conditions of triangular fuzzy number, i.e. the lower and upper values converge to one value at $r=1$. On the other hand figures 3-4, 7-8, 11-12, having trapezoidal curves indicate the verification of trapezoidal fuzzy number, i.e. the lower and upper values converge to two different values $r=1$. The overlapping of dotes and lines indicates closeness of the approximated and exact values which further verifies the accuracy of the proposed methods.

Figures 3, 11, 19 represent triangular curves of problems 1, 3 and 5, respectively, for 0.75 order of derivative i.e. $\beta=0.75$, calculated by Method 1and Method 2. And figures 4, 12, 20 show triangular plots for 0.85 order of derivative i.e. $\beta=0.85$, of example 1,3 and 5 , respectively, calculated by Method 1 and Method 2. The triangular curves indicate that the fractional order calculated by considered methods also satisfy conditions of triangular fuzzy numbers. Conversely, figures 7, 15, 23 represent trapezoidal curves for $\beta=0.75$ and figures $8,16,24$ trapezoidal curves for $\beta=0.85$, of examples 2 , 4 , and 6, respectively. Observably, fractional orders of these examples, calculated using Method 1 and Method 2, also converge to two different points, making a trapezoidal curve. From these figures it can be clearly resolved that the proposed methods are also applicable and efficient for fractional order derivatives. 


\section{Conclusions}

In this paper, advancement in Method 1 and Method 2 of Max-Min Improved Euler methods [29] for the fractional order has been made. The proposed methods are demonstrated through linear and nonlinear fractional differential equations with triangular and trapezoidal fuzzy initial values. Relatively, followings results are concluded:

- The proposed method converges to the exact solution more rapidly.

- Numerical results show that for smaller step size smaller error and hence better and accurate results are obtained.

- Table values and graphical plots for trapezoidal fuzzy initial values satisfy the definition of trapezoidal fuzzy number in section 3.1, i.e. results obtained from proposed methods approach to two different solutions for the upper and the lower solutions of the problems.

- Similarly, Table values and graphical plots for triangular fuzzy initial values satisfy the definition of triangular fuzzy number in section 3.2, i.e.results obtained from proposed method approach to one solution for the upper and the lower solutions of the problems.

- It is shown that the proposed method yielded accurate approximations.

The computations corresponding to the problems have been performed using Mathematica 9. Accordingly, the proposed methods are efficiently applicable for the fuzzy fractional differential equations of both linear and nonlinear problems. It can be noticed that presented methods show an easy and efficient way of acquiring accurate solution. 


\section{Appendix}

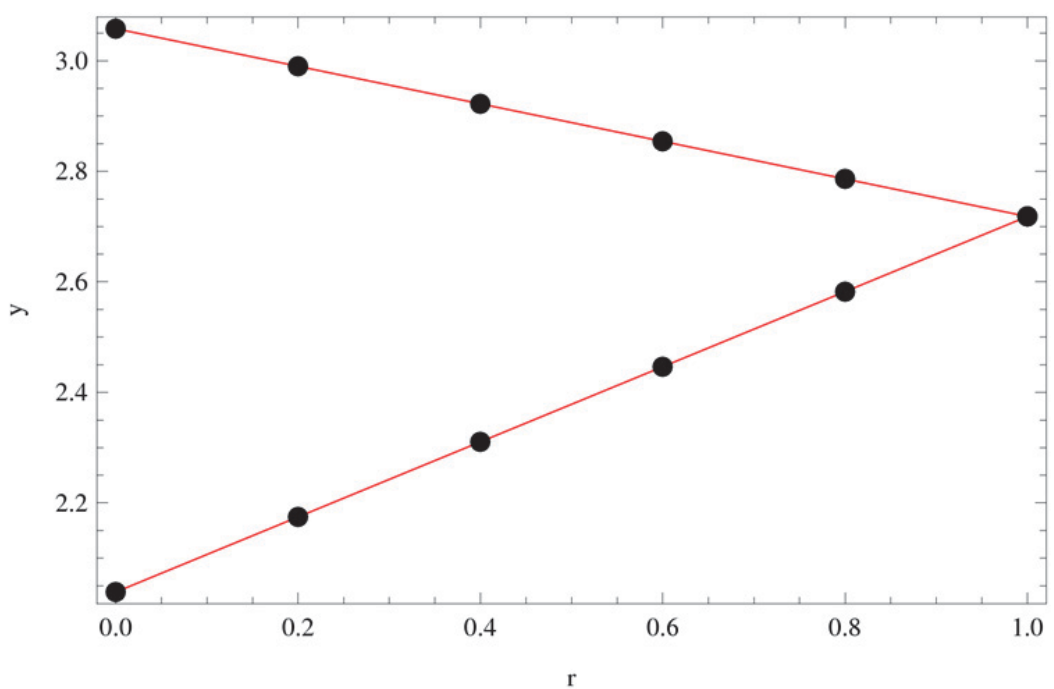

Figure 1. Comparison between exact and calculated values of problem 1 by Method 1 with $\beta=1$ and $h=0.001$ at $x=1$. (Exact represented by line and calculated represented by dots)

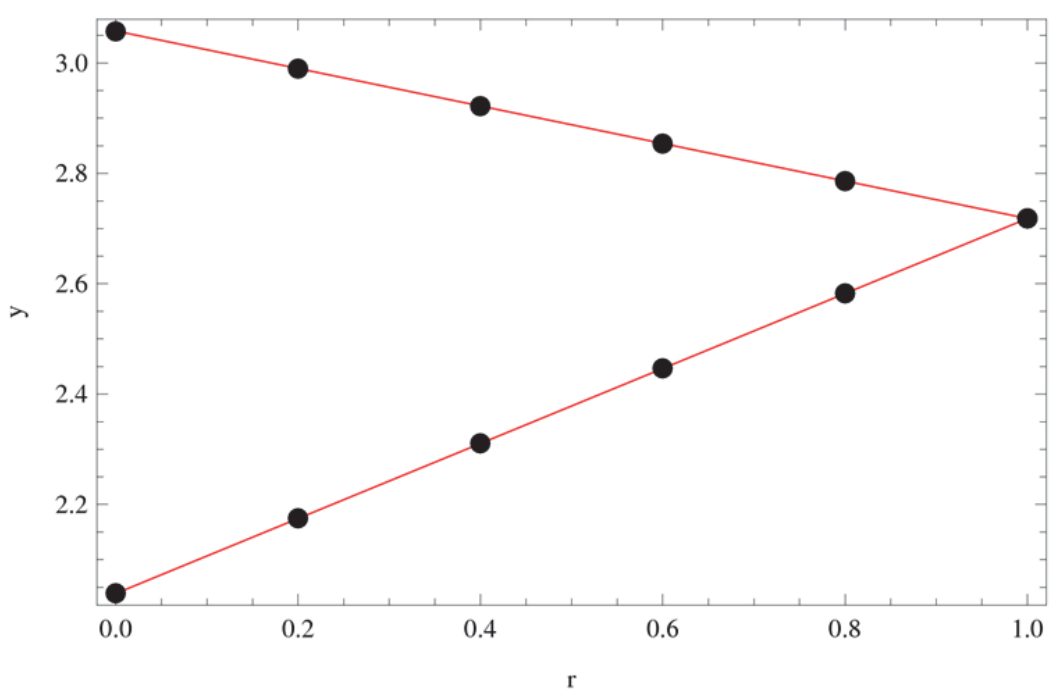

Figure 2. Comparison between Exact and calculated values of problem 1 by Method 2 with $\beta=1$ and $h=0.001$ at $x=1$. (Exact represented by line and calculated represented by dots) 


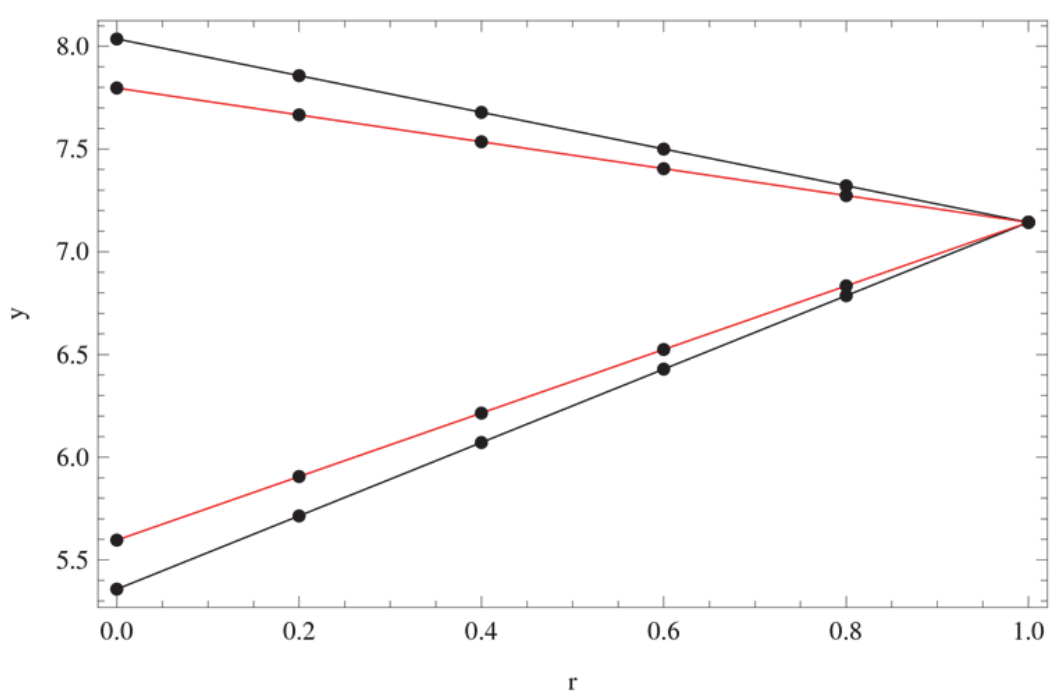

Figure 3. Comparison between Method 1and Method 2 of problem 1 with $\beta=0.75$ and $h=0.1$ at $x=1$. (Black for Method 1, Red for Method 2)

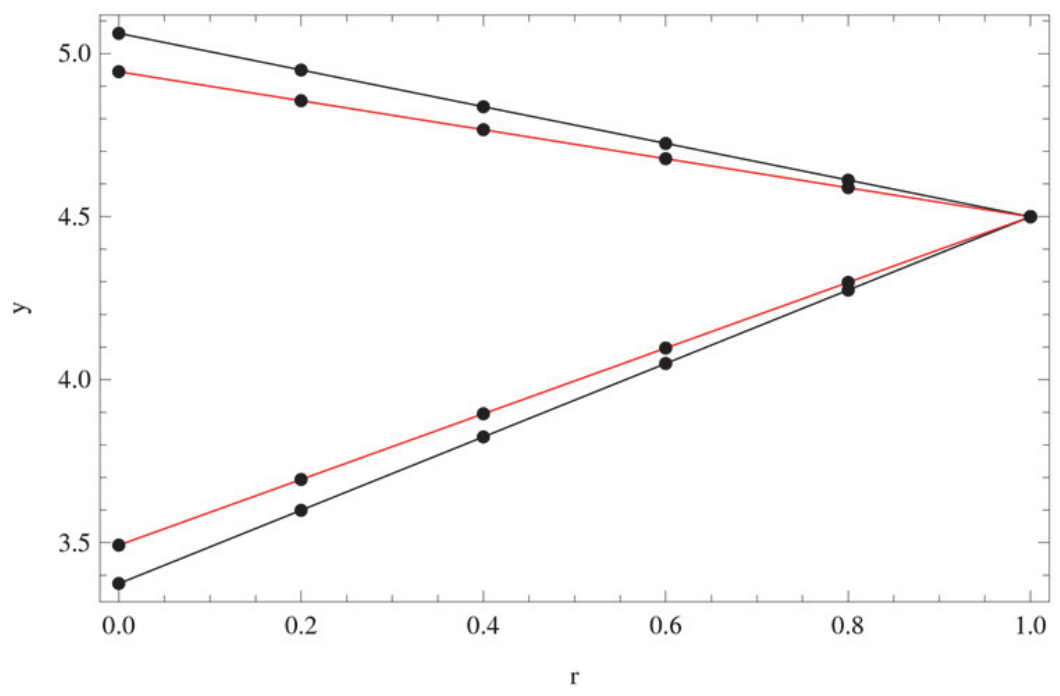

Figure 4. Comparison between Method 1and Method 2 of problem 1 with $\beta=0.85$ and $h=0.1$ at $x=1$. (Black for Method 1, Red for Method 2) 
Khan N. A., Razzaq O. A., Riaz F.

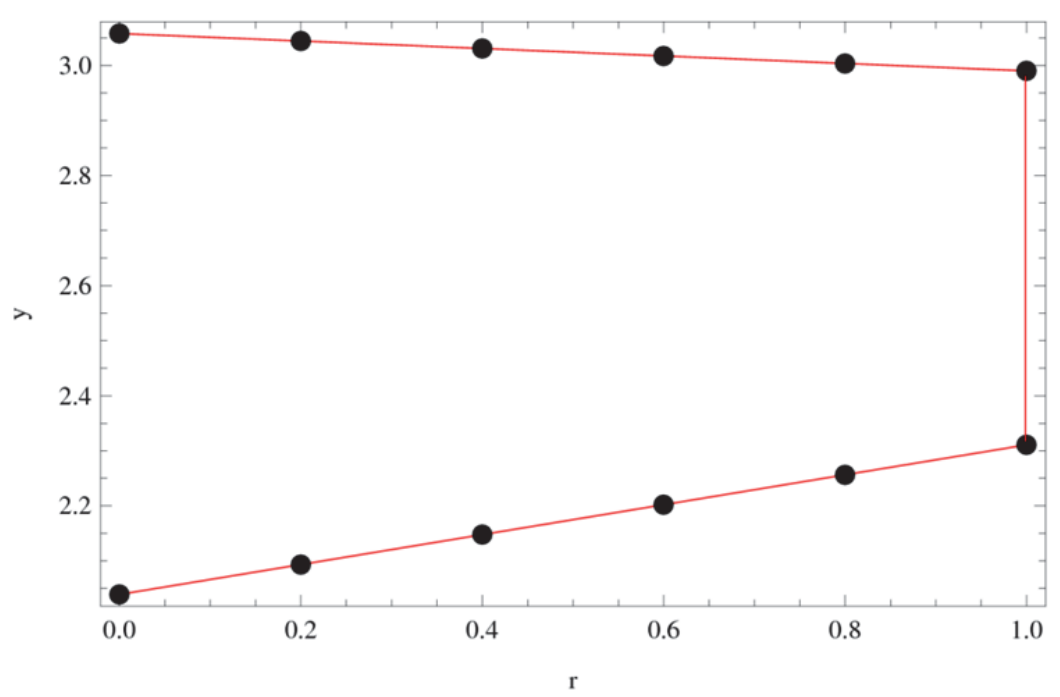

Figure 5. Comparison between exact and calculated values of problem 2 by Method 1 with $\beta=1$ and $h=0.001$ at $x=1$. (Exact represented by line and calculated represented by dots)

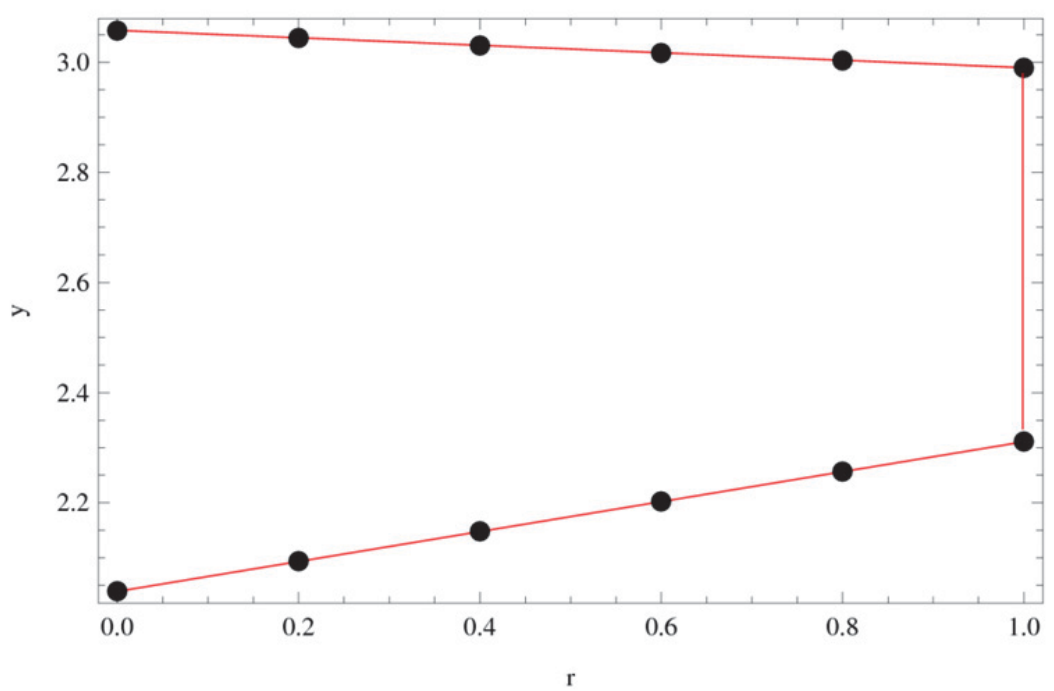

Figure 6. Comparison between Exact and calculated values of problem 2 by Method 2 with $\beta=1$ and $h=0.001$ at $x=1$. (Exact represented by line and calculated represented by dots) 
Numerical Simulations For Solving Fuzzy ...

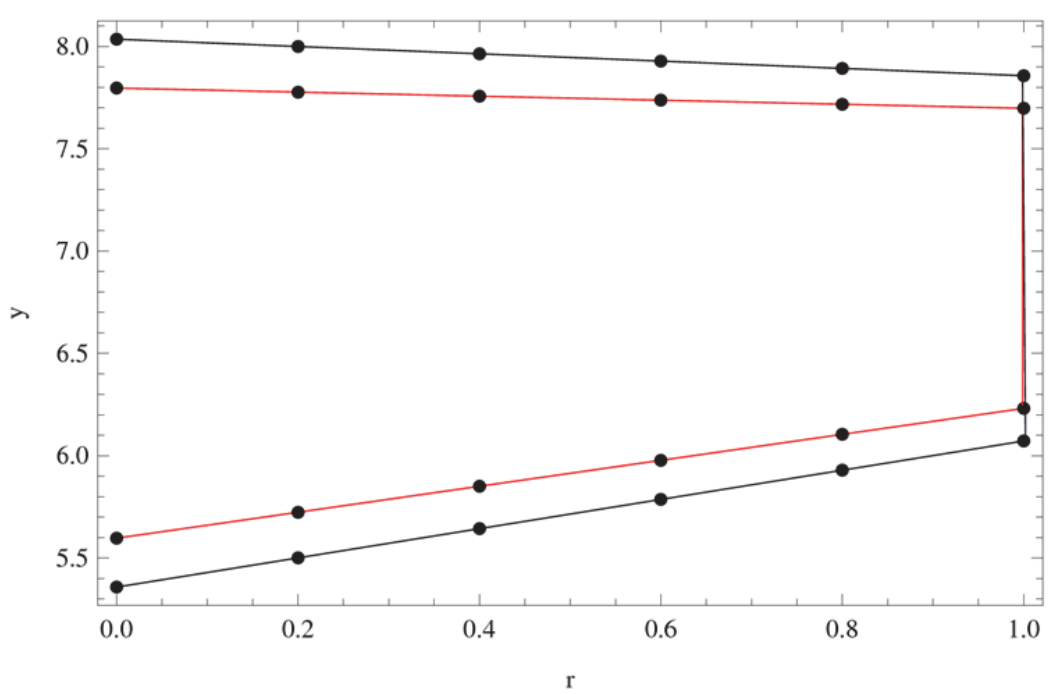

Figure 7. Comparison between Method 1and Method 2 of problem 2 with $\beta=0.75$ and $h=0.1$ at $x=1$. (Black for Method 1, Red for Method 2)

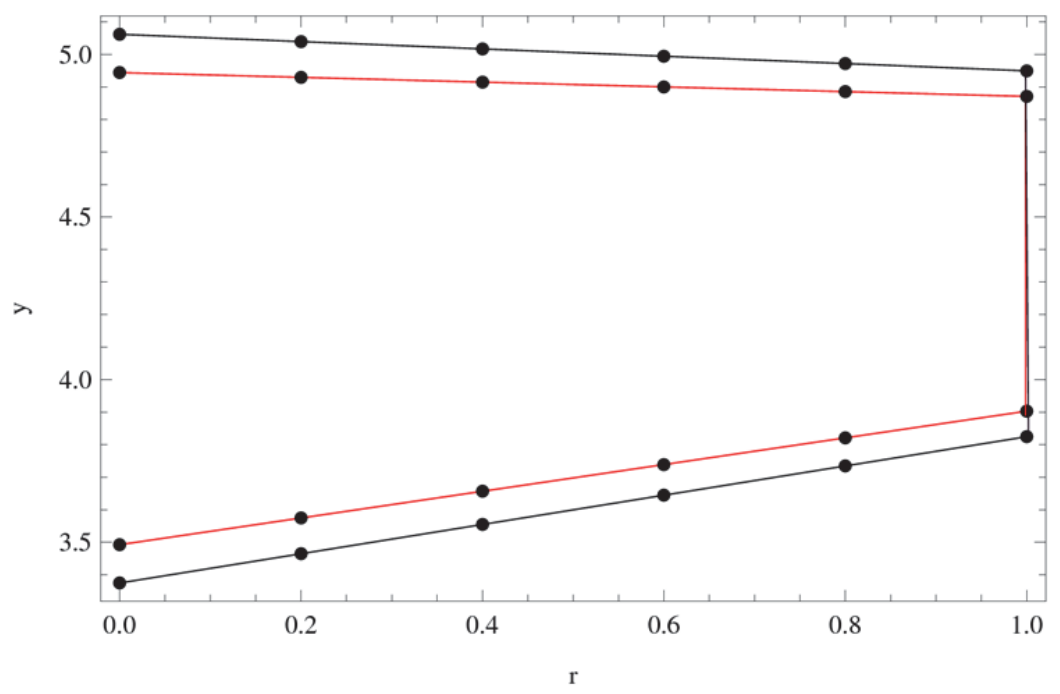

Figure 8. Comparison between Method 1and Method 2 of problem 2 with $\beta=0.85$ and $h=0.1$ at $x=1$. (Black for Method 1, Red for Method 2) 
Khan N. A., Razzaq O. A., Riaz F.

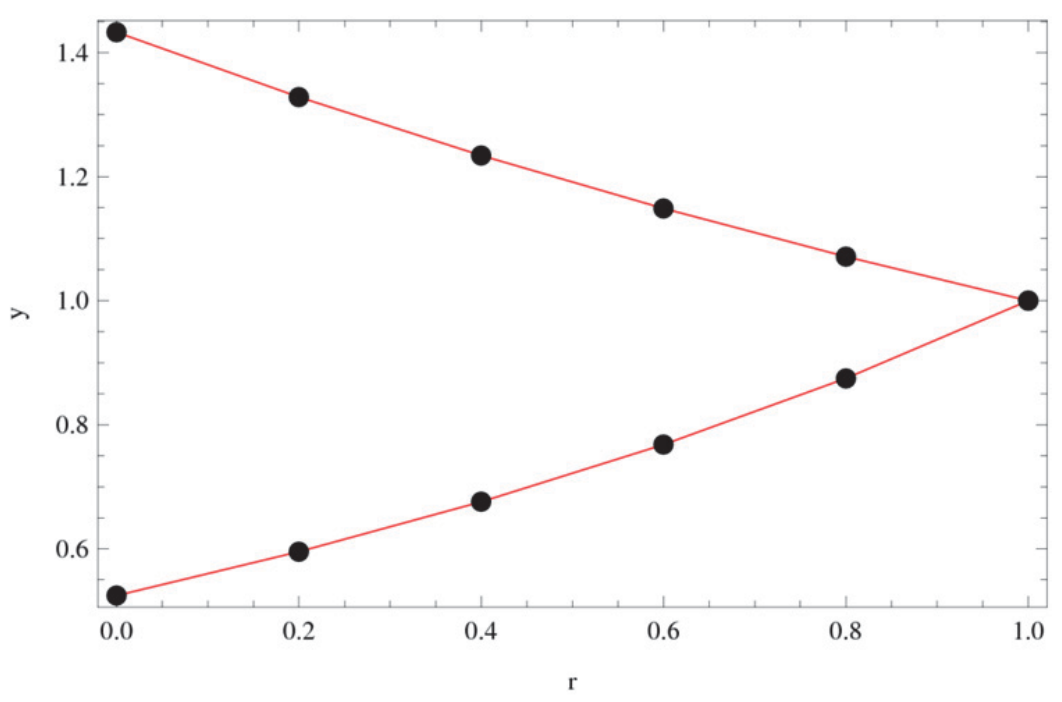

Figure 9. Comparison between Exact and calculated values of problem 3 by Method 1 with $\beta=1$ and $h=0.001$ at $x=1$. (Exact represented by line and calculated represented by dots)

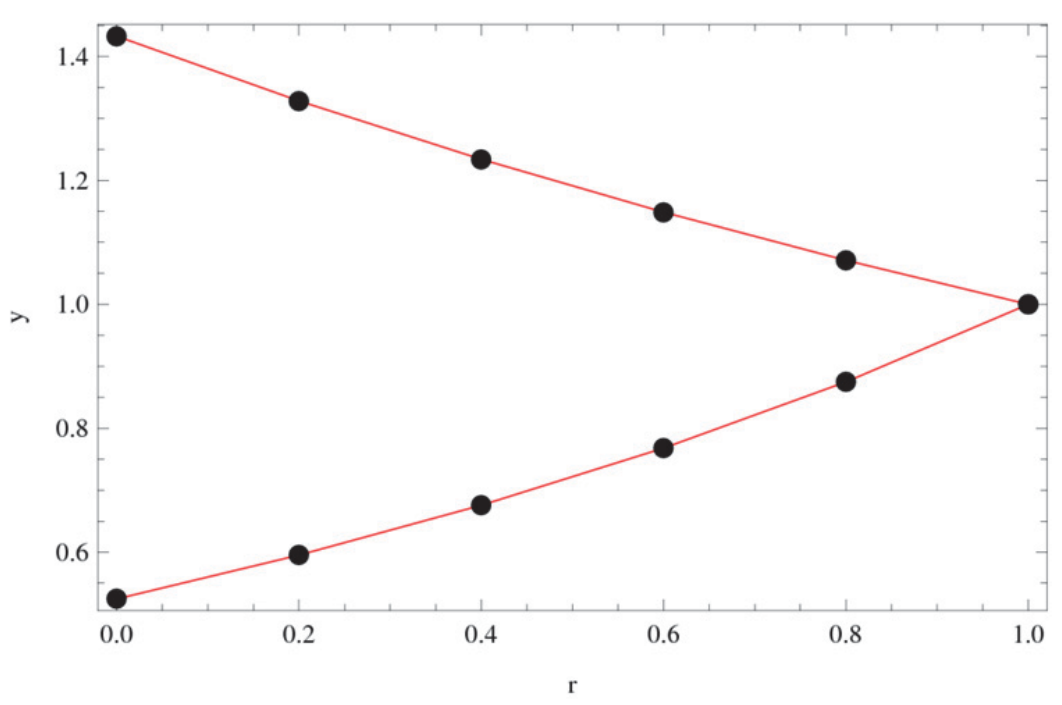

Figure 10. Comparison between Exact and calculated values of problem 3 by Method 2 with $\beta=1$ and $h=0.001$ at $x=1$. (Exact represented by line and calculated represented by dots) 


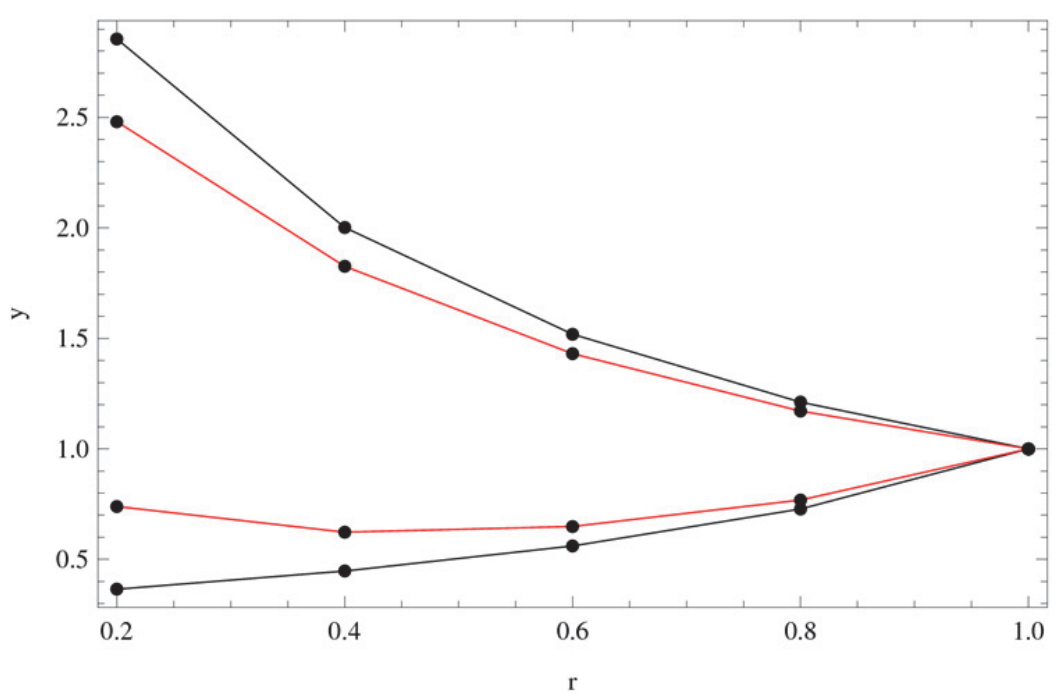

Figure 11. Comparison between Method 1and Method 2 of problem 3 with $\beta=0.75$ and $h=0.1$ at $x=1$. (Black for Method 1, Red for Method 2)

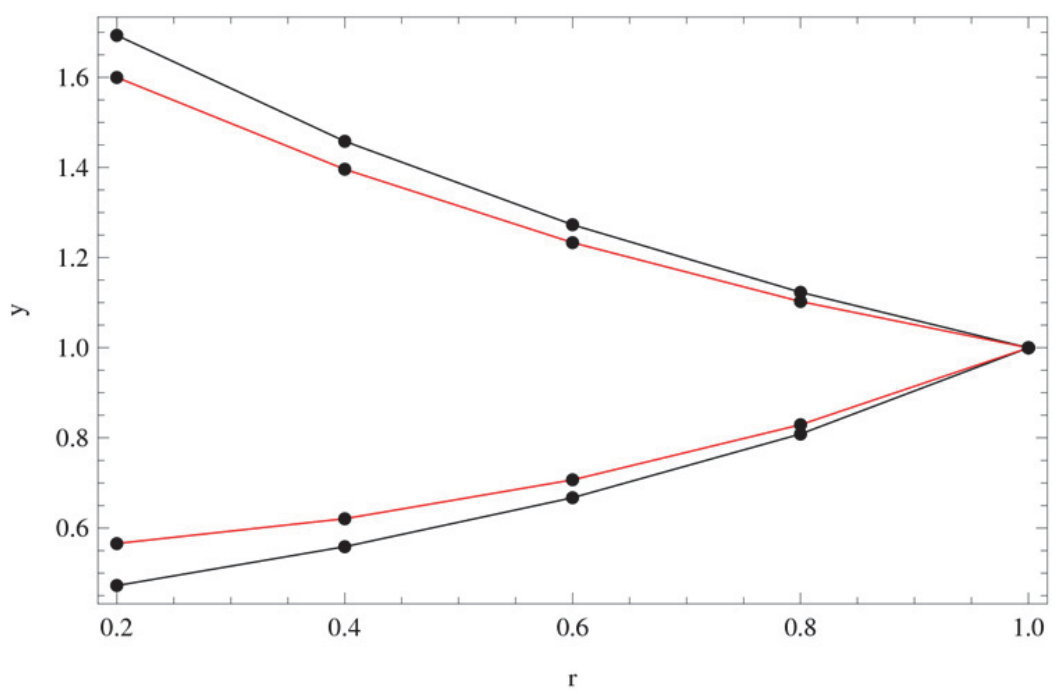

Figure 12. Comparison between Method 1and Method 2 of problem 3 with $\beta=0.85$ and $h=0.1$ at $x=1$. (Black for Method 1, Red for Method 2) 
Khan N. A., Razzaq O. A., Riaz F.

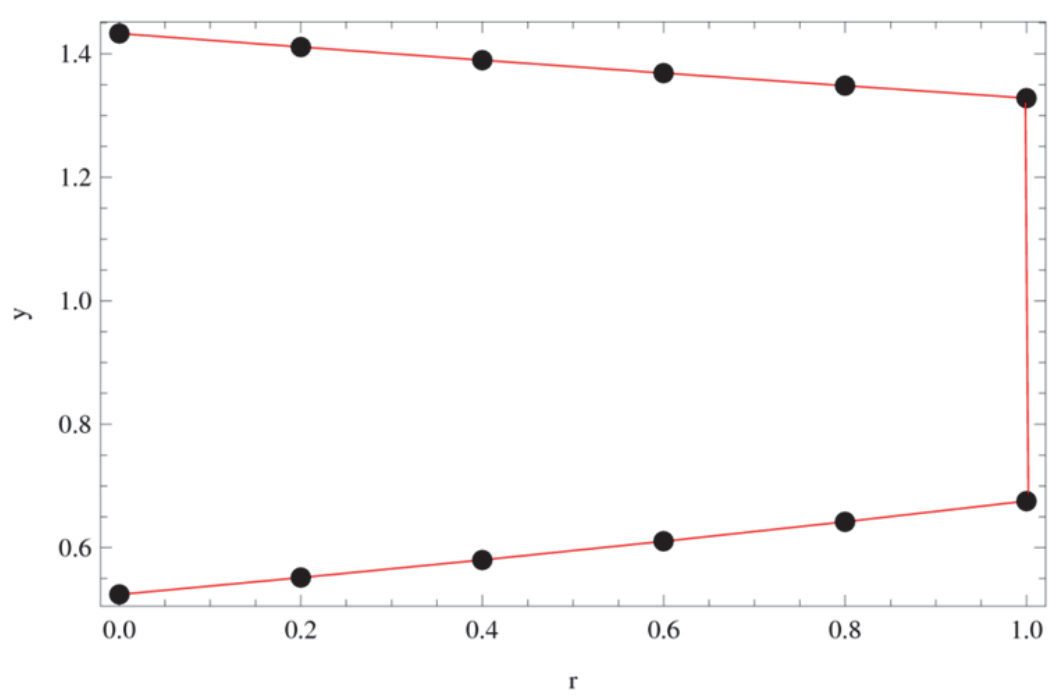

Figure 13. Comparison between Exact and calculated values of problem 4 by Method 1 with $\beta=1$ and $h=0.001$ at $x=1$. (Exact represented by line and calculated represented by dots)

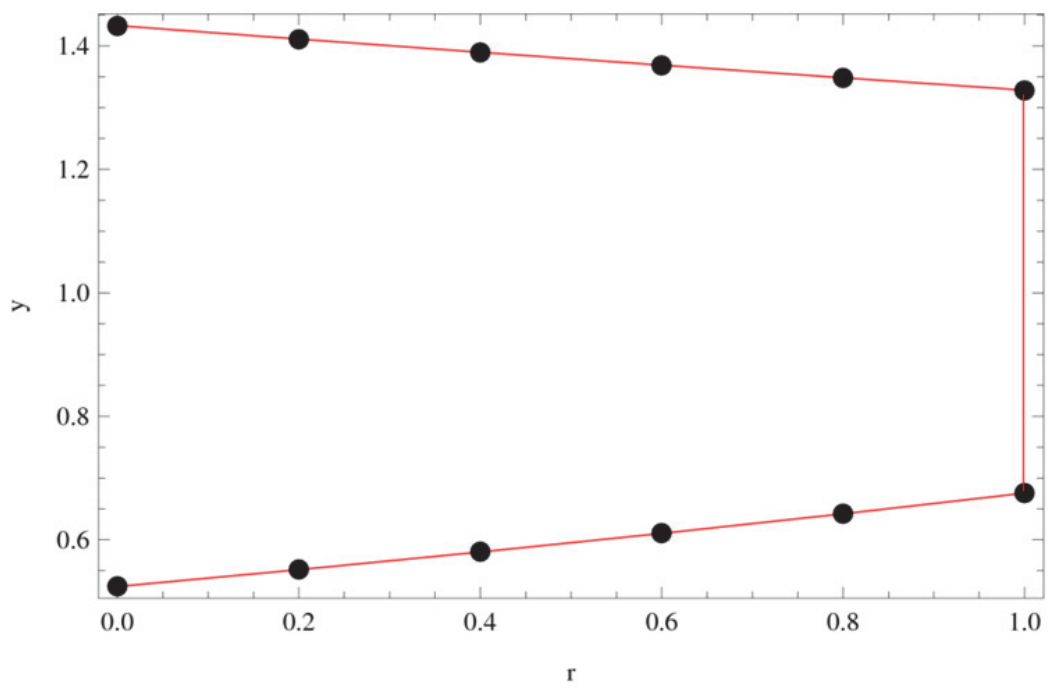

Figure 14. Comparison between Exact and calculated values of problem 4 by Method 2 with $\beta=1$ and $h=0.001$ at $x=1$. (Exact represented by line and calculated represented by dots) 
Numerical Simulations For Solving Fuzzy ...

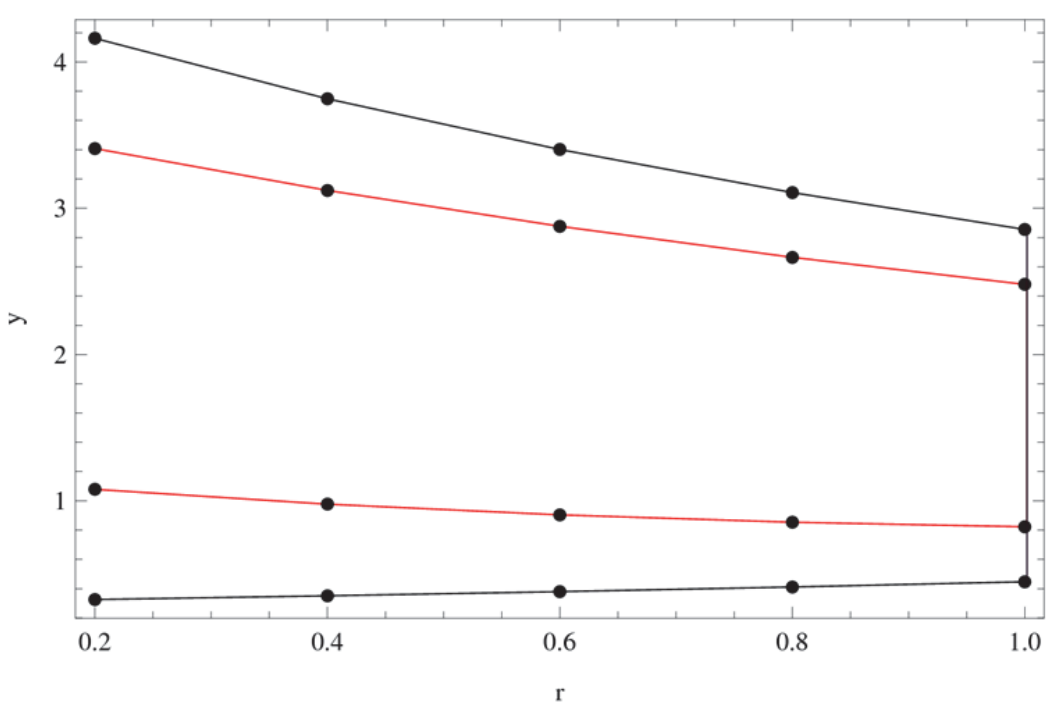

Figure 15. Comparison between Method 1and Method 2 of problem 4 with $\beta=0.75$ and $h=0.1$ at $x=1$. (Black for Method 1, Red for Method 2)

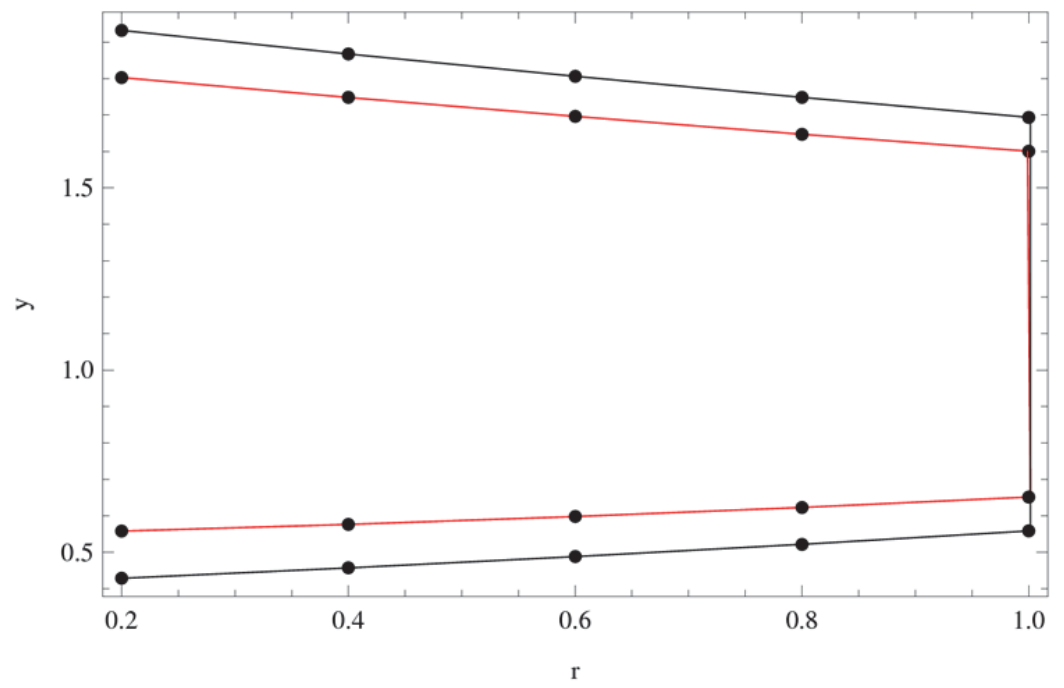

Figure 16. Comparison between Method 1and Method 2 of problem 4 with $\beta=0.85$ and $h=0.1$ at $x=1$. (Black for Method 1, Red for Method 2) 
Khan N. A., Razzaq O. A., Riaz F.

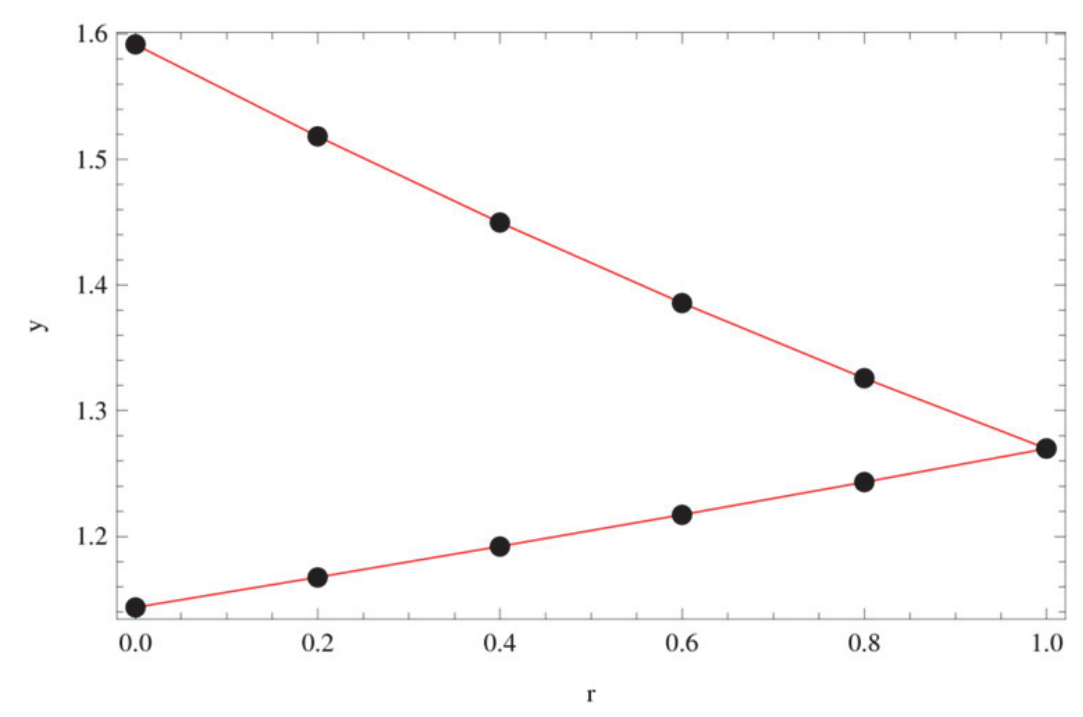

Figure 17. Comparison between Exact and calculated values of problem 5 by Method 1 with $\beta=1$ and $h=0.001$ at $x=1$. (Exact represented by line and calculated represented by dots)

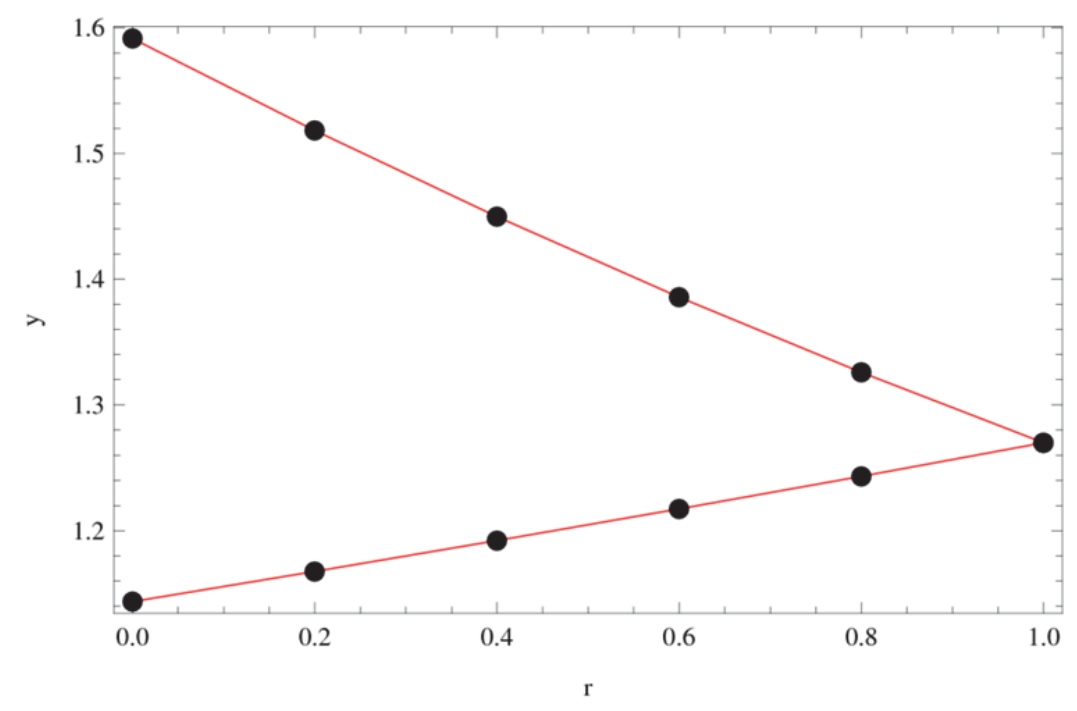

Figure 18. Comparison between exact and calculated values of problem 5 by Method 2 with $\beta=1$ and $h=0.001$ at $x=1$. (Exact represented by line and calculated represented by dots) 


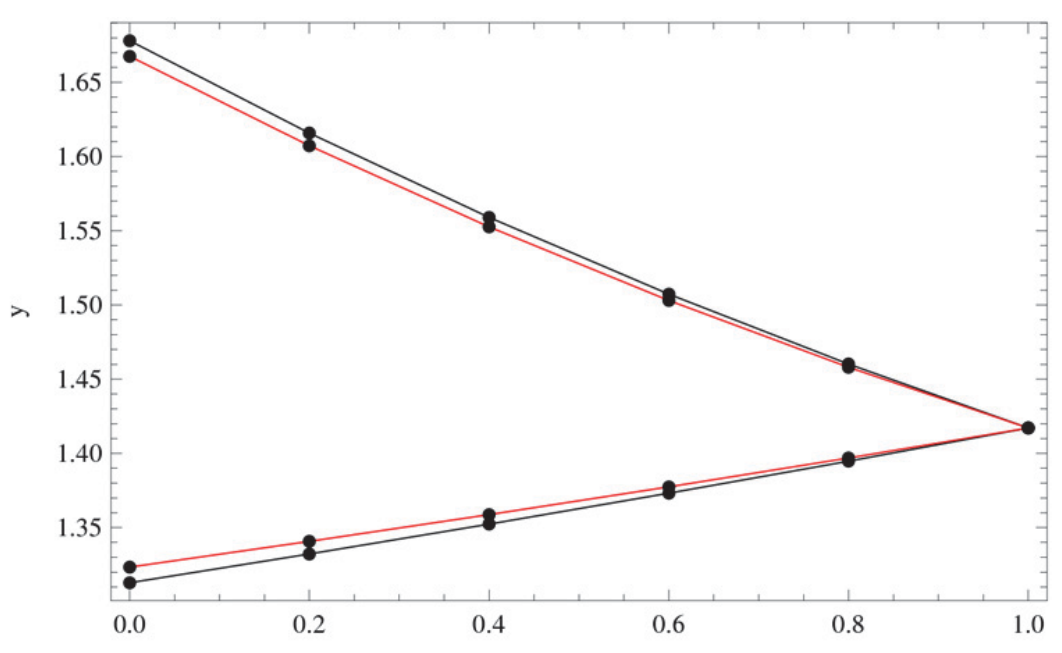

Figure 19. Comparison between Method 1and Method 2 of problem 5 with $\beta=0.75$ and $h=0.1$ at $x=1$. (Black for Method 1, Red for Method 2)

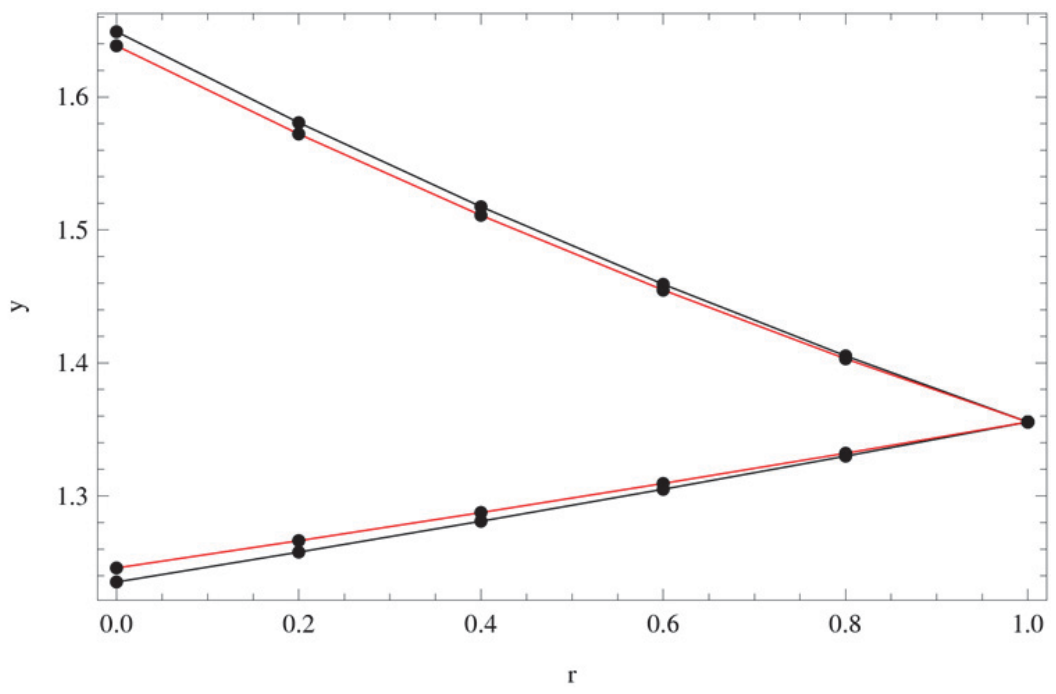

Figure 20. Comparison between Method 1and Method 2 of problem 5 with $\beta=0.85$ and $h=0.1$ at $x=1$. (Black for Method 1, Red for Method 2) 
Khan N. A., Razzaq O. A., Riaz F.

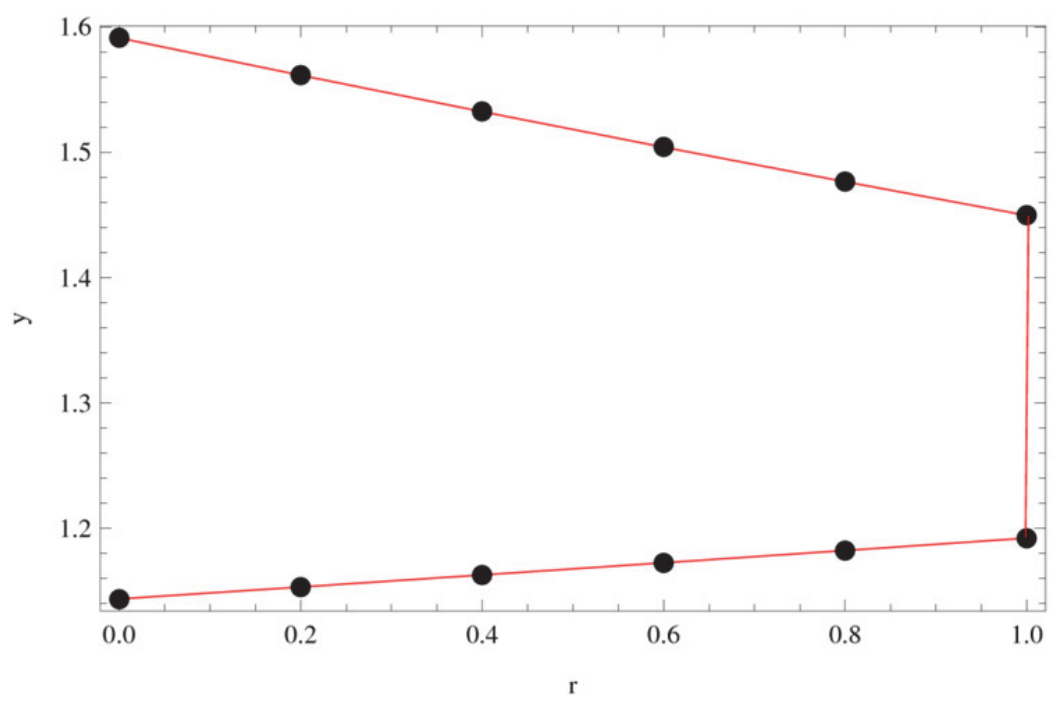

Figure 21. Comparison between Exact and calculated values of problem 6 by Method 1 with $\beta=1$ and $h=0.001$ at $x=1$. (Exact represented by line and calculated represented by dots)

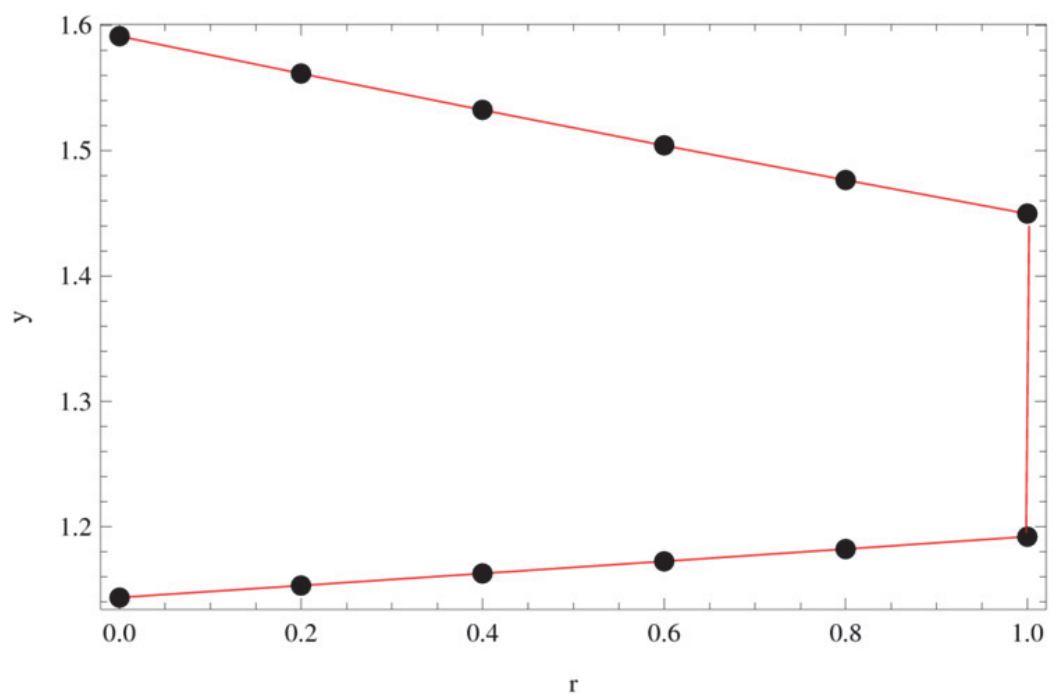

Figure 22. Comparison between Exact and calculated values of problem 6 by Method 2 with $\beta=1$ and $h=0.001$ at $x=1$. (Exact represented by line and calculated represented by dots) 
Numerical Simulations For Solving Fuzzy ...

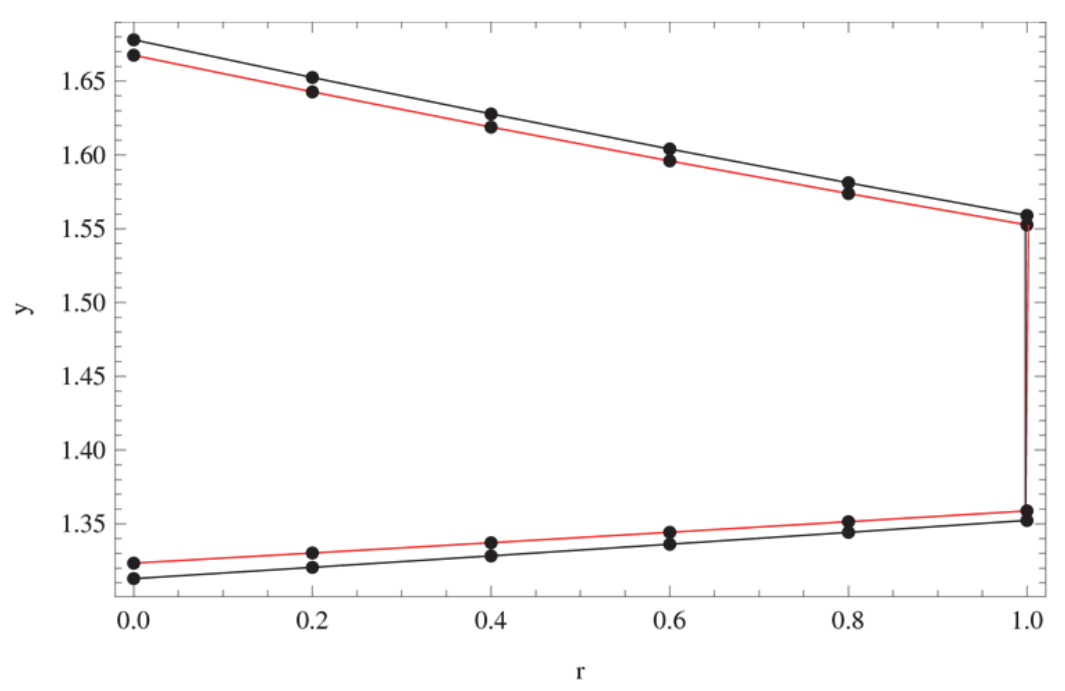

Figure 23. Comparison between Method 1 and Method 2 of problem 6 with $\beta=0.75$ and $h=0.1$ at $x=1$. (Black for Method 1, red for Method 2)

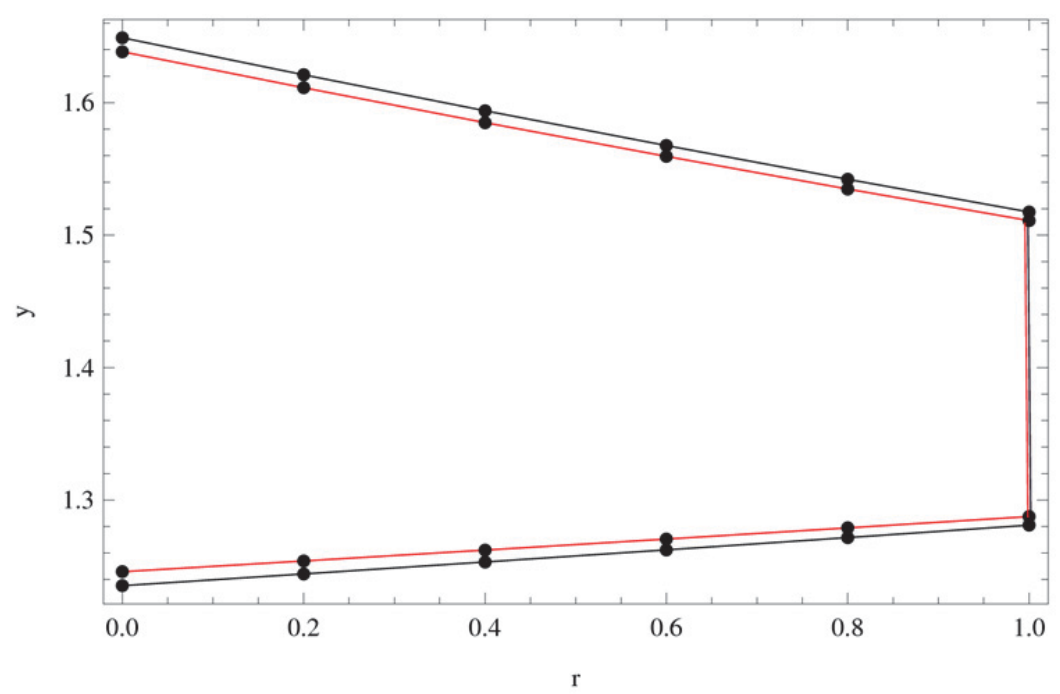

Figure 24. Comparison between Method 1and Method 2 of problem 6 with $\beta=0.85$ and $h=0.1$ at $x=1$. (Black for Method 1, red for Method 2) 


\section{References}

1. Kilbas AA, Srivastava HM, Trujillo JJ, 2006, Theory and applications of fractional differential equations, San Diego, Elsevier.

2. Bagley RL, 1990, On the fractional order initial value problem and its engineering applications, in Fractional Calculus and Its Applications, K. Nishimoto, Ed., College of Engineering, Nihon University, Tokyo, Japan 12-20.

3. Podlubny I, 1990, Fractional differential equations, Academic Press, San Diego.

4. Machado JT, Kiryakova V, Mainardi F, 2011, Recent history of fractional calculus. Communications in Nonlinear Science and Numerical Simulation 16: 1140 1153.

5. Diethelm K, Ford NJ, 2002, Analysis of fractional differential equations. Journal of Mathematical Analysis and Applications 265: 265-322.

6. Das S, 2008, Functional fractional calculus for system identification and controls. New York: Springer.

7. Adda FB, Cresson J, 2005, Fractional differential equations and the Schrodinger equation. Applied Mathematics and Computation 161: 323-345.

8. Fellah ZEA, Depollier C, Fellah M, 2002, Application of fractional calculus to the sound waves propagation in rigid porous materials: validation via ultrasonic measurements. Acta Acustica united with Acustica, 88: 34-39.

9. Rahimy M, 2012, Applications of fractional differential equations. Applied Mathematical Sciences 4: 2453-2461.

10. Khan NA, Mahmood A, Ara A, Khan NA, 2012, Analytical study of nonlinear fractional order Integro-differential equation: Revisit Volterra's population model. International Journal of Differential Equations: Article ID 845945.

11. Ahmad B, Nieto JJ, 2009, Existence results for a coupled system of nonlinear fractional differential equations with three-point boundary conditions. Computer and Mathematics with Applications 58: 1838-1843.

12. Jumarie G, 2009, Table of some basic fractional calculus formulae derived from a improved Riemann-Liouville derivative for non-differentiable functions. Applied Mathematics Letters 22: 378-385.

13. Khan NA, Khan NU, Ara A, Jamil M, 2012, Approximate analytical solutions of fractional reaction-diffusion equations. Journal of King Saud University - Science 24: 111-118.

14. Khan NA, Ara A, Mahmood A, 2012, Numerical solutions of time fractional Burger equations: a comparison between generalized transformation technique with homotopy perturbation method. International Journal of Numerical Method and Heat Fluid Flow 22: 175-93.

15. Jafari H, Tajadodi H, Matikolai SAH, 2011, Homotopy perturbation pade technique for solving fractional Riccati differential equations. The International Journal of Nonlinear Sciences and Numerical Simulation 11: 271-276.

16. Jafari H, Tajadodi H, Nazari H, Khalique CM, 2011, Numerical solution of nonlinear Riccati differential equations with fractional order. The International Journal of Nonlinear Sciencesand Numerical Simulation 11: 179-182.

17. Araya D, Lizama C, 2008, Almost automorphic mild solutions to fractional differential equations. Nonlinear Analysis 69: 3692-3705. 
18. Khan NA, Ara A, Jamil M, 2011, An efficient approach for solving the Riccati equation with fractional orders. Computer and Mathematics with Applications 61: 2683-2689.

19. Abbasbandy S, Shirzadi A, 2010, Homotopy analysis method for multiple solutions of the fractional Sturm-Liouville problems. Numerical Algorithms 54: 521532 .

20. Khan NA, Jamil M, Ara A, Khan NU, 2011, On efficient method for system of fractional differential equations. Advances in Difference Equations: Article $\mathrm{ADE} / 303472$

21. Zadeh LA, 1965, Fuzzy sets. Information and Control 8: 338-353.

22. Zadeh LA, 1978, Fuzzy sets as a basis for a theory of possibility. Fuzzy Sets and Systems 1: 3-28.

23. Agarwal RP, Lakshmikantham V, Nieto JJ, 2010, On the concept of solution for fractional differential equations with uncertainty. Nonlinear Analysis 72: 28592862.

24. Ahmad MZ, Hasan MK, Abbasbandy S, 2013, Solving fuzzy fractional differential equations using Zadeh's extension principle. The ScientificWorld Journal 2013: Article ID 454969.

25. Arshad S, Lupulescu V, 2011, On the fractional differential equations with uncertainty. Nonlinear Analysis: Theory, Methods \&Applications 74: 3685-3693.

26. Allahviranloo T, Salahshour S, Abbasbandy S, 2012, Explicit solutions of fractional differential equations with uncertainty. Soft Computing 16: 297-302.

27. Salahshour S, Allahviranloo T, Abbasbandy S, 2012, Solving fuzzy fractional differential equations by fuzzy Laplace transforms. Communications in Nonlinear Science and Numerical Simulation 17: 1372-1381.

28. Alikhani R, Bahrami F, 2013, Global solutions for nonlinear fuzzy fractional integral and integro differential equations. Communications in Nonlinear Science and Numerical Simulation 18: 2007-2017.

29. Tapaswini S, Chakraverty S, 2012, A new approach to fuzzy initial value problem by improved Euler method. Fuzzy Information and Engineering 3: 293-312.

30. Ochoche A, 2008, Improving the improved Euler method for better performance on autonomous initial value problems. Leonardo Journal of Sciences 12: $57-66$.

31. Ma M, Friedman M, Kandel A, 1999, Numerical solutions of fuzzy differential equations. Fuzzy Sets and Systems 105: 133-138.

32. Mazandarani M, Kamyad AV, 2013, Improved fractional Euler method for solving fuzzy fractional initial value problem. Communications in Nonlinear Science and Numerical Simulation 18: 12-21.

33. Ahmad MZ, Hasan MK, Baets BD, 2013, Analytical and numerical solutions of fuzzy differential equations. Information Sciences 236: 156-167.

34. Allahviranloo T, Abbasbandy S, Salahshour S, Hakimzadeh A, 2011, A new method for solving fuzzy linear differential equations. Computing 92: 181-197.

35. Shokri J, 2007, Numerical solution of fuzzy differential equations. Applied Mathematical Sciences 1: 2231-2246.

36. Duraisamy C, Usha B, 2010, Another approach to solution of fuzzy differential equations by improved Euler's method. Proceedings of the International conference on Communication and Computational Intelligence-kongu Engineering College, Perundurai, Erode, TN, India: 52-55. 
37. Odibat Z, Momani S, 2008, An algorithm for the numerical solution of differential equations of fractional order. Journal of Applied Mathematics \& Informatics 26: 15--27.

38. Aminikhah H, Hemmatnezhad M, 2010, An efficient method for quadratic Riccati differential equation. Communication in Nonlinear Science and Numerical Simulation 15: 835--839.

39. Bede B, Gal SG, 2005, Generalizations of the differentiability of fuzzy-numbervalued functions with applications to fuzzy differential equations. Fuzzy Sets System 151: 581--599. 